\title{
Special Majorities Rationalized
}

\author{
ROBERT E. GOODIN AND CHRISTIAN LIST*
}

Complaints are common about the arbitrary and conservative bias of special-majority rules. Such complaints, however, apply to asymmetrical versions of those rules alone. Symmetrical special-majority rules remedy that defect, albeit at the cost of often rendering no determinate verdict. Here what is formally at stake, both procedurally and epistemically, is explored in the choice between those two forms of special-majority rule and simple-majority rule; and practical ways are suggested of resolving matters left open by symmetrical special-majority rules - such as 'judicial extrapolation' or 'subsidiarity' in a federal system.

The draft Constitution for Europe begins by invoking Pericles' Funeral Oration: 'Our Constitution ... is called a democracy because power is in the hands ... of the greater number'. But that is not quite true. Instead of rule purely by 'the greater number' - simple majority rule - the draft Constitution prescribes 'qualified majority' rule, with decisions of the European Council or Council of Ministers sometimes requiring the consent of as many as two-thirds of the member states, 'representing at least three fifths of the population of the Union'. 1

Such 'special' (or 'qualified' or 'super') majority requirements are not uncommon. ${ }^{2}$ It may take only a majority vote of both houses of the US Congress to declare war; but it takes a two-thirds majority to override a president's veto or three-fifths to close Senate debate. ${ }^{3}$ Increasing taxes requires the support of between three-fifths and three-quarters of legislators in many American states. ${ }^{4}$ Criminal verdicts must be unanimous, or nearly so. ${ }^{5}$

Super-majorities are sometimes seen as second-best forms of unanimity rules, employed where decisions ought ideally to be unanimous but where the costs of securing unanimity

* Social \& Political Theory and Philosophy Programs, RSSS, Australian National University, Canberra; Department of Government, London School of Economics, respectively. The authors are grateful for comments from John Dryzek, John Ferejohn, Dennis Mueller, Albert Weale and anonymous referees. This article was written while List was a Post-doctoral Fellow in the Social \& Political Theory Program, RSSS, Australian National University.

${ }^{1}$ Article 24 (pp. 5, 21) of Draft Treaty Establishing a Constitution for Europe, submitted to the European Council Meeting in Thessaloniki, 28 June 2003. CONV 820/1/03 REV 1, http://european-convention.eu.int/docs/ Treaty/cv00820-re01.en03.pdf (accessed 3 July 2003).

2 They are commonly set in the context of elaborate rules and procedures, with many of the decisions preceding the final vote being made by simple or even submajority rules; Adrian Vermeule, 'Submajority Rules: Forcing Accountability upon Majorities', Journal of Political Philosophy, 13 (2005), 74-98. Real-world decision rules are thus far more complex than the simple versions captured by formal models, here and elsewhere.

3 David R. Mayhew, 'Supermajority Rule in the U.S. Senate', PS: Political Science \& Politics, 36 (2002), 31-6.

4 Nearly a third of them, in fact. See Mandy Rafool, 'Which States Require a Supermajority to Raise Taxes?' National Conference of State Legislatures, March 1988, http://www.ncsl.org/programs/fiscal/suprmajr.htm (accessed 2 July 2003).

5 Ten-to-two verdicts are accepted in England and some American states: Jeffrey Abrahamson, We, the Jury (New York: Basic Books, 1994), p. 180. 
would be too high. ${ }^{6}$ The plain political fact is that the larger the majority required, the less likely it is to be secured.

Special-majority rules of the ordinary form leave existing arrangements in place unless there is some positive decision to change them. Hence, such rules have a powerful conservative bias. ${ }^{7}$ That is precisely their attraction, for those attracted to them.

Justifying special-majority rules thus appears to be largely a matter of justifying their conservative bias. Sometimes the bias seems justified. Most of us, for example, think it right that there should be a presumption of innocence in criminal trials, and that it should be hard to overcome that presumption. ${ }^{8}$ Usually, however, the bias is controversial. ${ }^{9}$ Sometimes there is not even a single status quo. ${ }^{10}$ Still other times, there seems no more reason for a decision procedure to be biased in one direction rather than the other. Civil trials, for example, are decided on the 'balance of probabilities'. The same standard of proof falls on both parties, rather than (as in criminal cases) one side having to establish its case 'beyond a reasonable doubt' and the other side winning by default otherwise.

The reluctance to let social decisions be determined by some arbitrary bias built into super-majority rules is captured in the complaint, 'It would be impossible to get the requisite majority for the status quo, either!" ${ }^{11}$ What is ordinarily taken to follow from that thought is that, when we have no grounds for 'a presumption one way or the other', then

${ }^{6}$ James M. Buchanan and Gordon Tullock, The Calculus of Consent (Ann Arbor: University of Michigan Press, 1962); Dennis C. Mueller, Public Choice III (Cambridge: Cambridge University Press, 2003), pp. $137-44$. Libertarians, for example, hold that people should not have authority exercised over them, or money taken from them, except with their consent: pragmatically, they concede, securing the consent of absolutely everyone to absolutely every enactment and absolutely every tax would preclude the efficient operation of government; still, they say, we should at least require substantial majorities for those purposes. Likewise with the criminal jury: ideally, we should convict someone only by a unanimous verdict; but where that cannot be obtained, at least we should demand a large (ten-to-two) majority. And likewise in amending constitutions: every state's consent was required in joining the union, so ideally every state's consent should be required in changing the terms of association; it may be impractical to demand unanimous consent to every amendment, but at least a large majority (three-quarters, in the United States) ought to be required.

7 William F. Samuelson and Richard Zeckhauser, 'Status Quo Bias in Decision Making', Journal of Risk and Uncertainty, 1 (1988), 7-59.

${ }^{8}$ Louis Kaplow, 'The Value of Accuracy in Adjudication: An Economic Analysis', Journal of Legal Studies, 23 (1994), 307-401.

${ }^{9}$ Libertarians may think there should be a presumption in favour of letting people do as they please, particularly with their own money, and that it should be hard for government to interfere with that. Others demur. Economists may think that there should be a presumption in favour of sticking to our long-term economic objectives, rather than succumbing to short-term temptations, and that central bankers operate according to super-majority rules to ensure that. Others demur, recalling with Keynes that 'in the long term we are all dead'. Regulators may think that drugs should be allowed on the market only once they have been proven conclusively to be safe and effective. Patients denied the therapeutic benefits of those drugs in the interim might once again demur. See Ernesto Dal Bó, 'Committees and Supermajority Voting: Balancing Commitment and Flexibility' (Discussion Paper No. 132, Department of Economics, University of Oxford, 2002, http://www.wadham.ox.ac.uk/ edalbo (accessed 3 July 2003); and Sam Peltzman, 'An Evaluation of Consumer Protection Legislation: The 1962 Drug Amendments', Journal of Political Economy, 81 (1973), 1049-91.

${ }^{10}$ Think of heterogeneous federations like the European Union: the status quo varies from one member state to the next; picking out any one of them as 'the' status quo to serve as the baseline from which any EU-wide policy making must proceed would be arbitrary.

11 Brian Barry, Political Argument (London: Routledge \& Kegan Paul, 1965), pp. 244, 312-16. As Rae puts it, 'If all outcomes were subject to unanimity, then we would risk [being in] the position in which we both refused to change policy and refused to keep it the same'; Douglas W. Rae, 'The Limits of Consensual Decision', American Political Science Review, 69 (1975), 1270-94, at p. 1279. 
we must 'surely abide by a simple majority'. ${ }^{12}$ To implement a super-majority rule in such cases would be to privilege, arbitrarily, whichever outcome is identified as the default option.

That problem of arbitrariness derives from the asymmetry of the familiar sorts of special-majority rules. One option is identified as the 'default option': it prevails if the other option does not secure the requisite 'special majority'. ${ }^{13}$ The default option can thus prevail without the support of anything approaching the requisite 'special majority', whereas the other option can prevail only if it does have such support. So ordinary special-majority rules are actually Asymmetrical Special-Majority rules (although they are not usually so labelled); and that asymmetry is the source of the complaint made above.

There is, however, an alternative way of specifying a special-majority rule without privileging any option. Remove the asymmetry. Under a Symmetrical Special-Majority rule, the same special majority of votes is required to install either option as the social decision. If neither option has such a special majority, then no option is chosen. This rule is symmetrical in holding each option to the same standard; no option is ever installed by default. This article elaborates this version of special-majority voting, after first having mapped the logical space in which all (simple and special) versions of majority rule are situated.

Formally, the great difference between Symmetrical and Asymmetrical SpecialMajority rules is this: if no option receives the requisite special majority, then under a Symmetrical rule no option is chosen, whereas under an Asymmetrical one the default option is chosen. This formal difference may matter materially. In Scotland, juries can return a verdict of 'convicted', 'acquitted' or 'case not proven'; and between those last two options there is a world of difference, the difference between full exoneration and lingering suspicion.

Of course, something always happens (or does not happen) as a result of any social decision, including the decision under a Symmetrical Special-Majority rule that 'no option is chosen'. In the case of the Scottish 'case not proven' verdict, the accused goes free, just as she would have done after a full-blown acquittal. Thus, it might be objected that there is no pragmatic difference between Symmetrical Special-Majority rules and Asymmetrical ones. Some outcome is always, de facto, the default outcome that will obtain in the absence of a special majority for doing something else.

But that conclusion would be mistaken. One reason has already been noted. Under a Symmetrical Special-Majority rule that outcome's status is merely de facto, whereas an Asymmetrical rule anoints some default outcome as de jure 'socially chosen' in such circumstances. And as noted, being set free de facto (because the case was 'not proven') is importantly different from being set free de jure (because you were acquitted).

There is another even more important reason why Symmetrical and Asymmetrical Special-Majority rules are different, explored at length below. Instead of specifying some option as the default outcome, as Asymmetrical Special-Majority rules do, Symmetrical

${ }^{12}$ Barry, Political Argument, p. 312.

${ }^{13}$ Or, more generally, if 'no other option' secures the requisite majority: but for simplicity we will confine our discussion here to the two-option case. The status quo is typically identified as the default option, but logically any option could be so designated. Specifying one option as the 'default' which wins (even if no one votes for it!), so long as no other option gets the requisite 'special majority', is just one case within the larger class of majoritarian-type rules which hold different options to different ('super', 'simple', or 'sub') 'majoritarian' standards. See Appendix I. 
Special-Majority rules can be supplemented with some alternative decision procedure that can be employed when no option receives the requisite special majority. ${ }^{14}$ Propositions that are 'not decided' by Symmetrical Special-Majority voting in one forum can be shifted to some other forum for resolution: to the House of Representatives, for example, in the case of a deadlock in Electoral College voting for the US president.

This suggestion is crucial in rescuing Symmetrical Special-Majority rules from the greatest worry that might surround them. Whereas the bugbear of Asymmetrical Special-Majority rules is arbitrariness of the default option, the bugbear of Symmetrical Special-Majority rules is that they may leave too much open. Sometimes, of course, things can be left open: no social decision is immediately required. But for those matters that cannot be left open, we propose that some supplementary mechanism can be used for settling things that Symmetrical Special-Majority voting cannot. We discuss the problem of breaking ties in Section IV and sketch some proposals in Section V.

We precede those practical considerations with some formal ones on those two alternative forms of special-majority voting. Democratic decision procedures can, broadly, be defended either on grounds of their procedural (fairness) merits or on grounds of their epistemic truth-tracking capacities or both. ${ }^{15}$ We analyse the formal characteristics of the two forms of special-majority voting, first from a procedural perspective (Section I) and then from an epistemic one (Section II), comparing both forms of special-majority rule with simple majority rule. The procedural standards are variations on those that Kenneth May famously showed to characterize Simple-Majority Voting itself. ${ }^{16}$ The epistemic standards are of a Bayesian sort, growing out of related work on the Condorcet Jury Theorem. ${ }^{17}$

We identify a 'trilemma', in both the procedural and epistemic realms. In each, there are three properties we might like a voting rule to display, but any given rule can display at most two of them at once. ${ }^{18}$ Our choice among voting rules - Simple-Majority rule, Asymmetrical Special-Majority rule or Symmetrical Special-Majority rule - depends on which of the three desiderata we are prepared to sacrifice. What is at stake in this choice is summarized in Section III.

Each of the formal discussions in this article is preceded by an informal statement of the issues involved. Furthermore, the formal discussions themselves are of a relatively accessible sort. Proofs appear in the appendices.

\footnotetext{
14 This might not always work. We may sometimes have good grounds for thinking that some procedure or forum is the uniquely correct one for deciding a certain issue, and think it would be wrong to let any other decide it. Or the other procedure or forum might yield no determinate outcome either. Still, even if the procedure or forum-shifting trick will not always necessarily work to settle matters left undecided by a Symmetrical Special-Majority rule, it might nevertheless go some distance towards assuaging those concerns.

15 Christian List and Robert E. Goodin, 'Epistemic Democracy: Generalizing the Condorcet Jury Theorem', Journal of Political Philosophy, 9 (2001), 276-306.

${ }^{16}$ Kenneth O. May, 'A Set of Independent, Necessary and Sufficient Conditions for Simple Majority Decision', Econometrica, 20 (1952), 680-4.

17 Robert E. Goodin, 'The Paradox of Persisting Opposition', Politics, Philosophy and Economics, 1 (2002), 109-46; Christian List, 'On the Significance of the Absolute Margin', British Journal for the Philosophy of Science, 55 (2004), 521-44; Christian List, 'The Epistemology of Special Majority Voting', Social Choice and Welfare (forthcoming).

${ }^{18}$ This is different from standard social-choice-theoretic impossibility results, in so far as those typically pertain to choices over more than two options. Our results, in contrast, show the impossibility of simultaneously satisfying certain desiderata even in two-option choices.
} 


\section{PROCEDURAL PROPERTIES OF SIMPLE AND SPECIAL-MAJORITY RULES}

Procedurally, the great attraction of democratic decision rules is that they embody a regime of 'fair equality' among participants in making collective decisions. ${ }^{19}$ No individual is privileged over any other. Moreover, under simple-majority voting - the paradigmatic democratic decision rule - no option is privileged over any other. An option is socially chosen or not, just depending on how many votes it gets, not on what option it is and not on who voted for it.

These criteria of 'fair equality' have been formalized in the social choice literature. There, May's Theorem 'is deservedly considered a minor classic' ${ }^{20}$ In a literature replete with negative (impossibility) results, May's Theorem tells us what positively can be said in favour of Simple-Majority rule. It shows that Simple-Majority rule - and it alone among all decision procedures - simultaneously satisfies four conditions, each of which seems independently desirable on democratic grounds.

Here we assess both forms of special-majority rule against analogous conditions. To foreshadow our conclusions: both forms of special-majority rule require a relaxation of one of those conditions, but different ones. Symmetrical Special-Majority rules relax the responsiveness condition (permitting more ties), Asymmetrical ones the symmetry condition. Which, if either, form of special-majority rule is attractive in a given context depends on whether we have grounds in that context for relaxing the relevant condition.

\section{An Informal Statement}

The conditions which May shows to be uniquely satisfied by Simple-Majority rule are stated formally in the next section. We here describe them informally and suggest why they are democratically appealing. We consider a social decision problem with two options (e.g. two candidates, or the acceptance or rejection of some proposition).

The first condition, 'universal domain', stipulates that the voting rule renders a decision (where a tie is a decision, too) for every logically possible combination - or 'profile' - of votes. ${ }^{21}$ This requirement is democratically compelling. A voting rule should be open to all possible combinations of votes that might be entered into it. If certain combinations of votes were rejected as inadmissible, they would be effectively disenfranchised. For technical simplicity, we assume that no voter is indifferent between the two options, but this assumption can in principle be relaxed.

The second condition, 'anonymity', stipulates that it does not matter who votes for what, ${ }^{22}$ all that matters is how many votes are cast for each option. The democratic appeal of this condition is obvious.

Just as 'anonymity' requires that all voters be treated equally, so the third condition, 'symmetry', requires that all options be treated equally. ${ }^{23}$ Again, it seems a democratically appealing requirement that a given combination of votes for one option should yield the

19 Charles R. Beitz, Political Equality (Princeton, N.J.: Princeton University Press, 1989).

${ }^{20}$ Brian Barry and Russell Hardin, eds, Rational Man and Irrational Society? (Beverley Hills, Calif.: Sage, 1982), p. 298. See also: Mueller, Public Choice III, pp. 133-6; R. Duncan Luce and Howard Raiffa, Games and Decisions (New York: Wiley. 1957), pp. 357-8; and Amartya Sen, Collective Choice and Social Welfare (San Francisco: Holden-Day, 1970), pp. 68, 70-1.

${ }^{21}$ May calls this condition 'decisiveness'.

${ }^{22}$ May calls this condition 'equality'.

23 May calls this condition 'neutrality'. 
same decision on that option that it would yield on another option if it were for that other option.

'Anonymity' and 'symmetry', 'taken together ... embody an interpretation of the basic idea of popular will theories of political fairness - any fair method for aggregating individual preferences should treat each person's preference equally' ${ }^{24}$ They embody the principle that each citizen's 'opinion is at least as good as any other's'. ${ }^{25}$

May's fourth condition, 'positive responsiveness', can be split into two conditions, 'monotonicity' and 'one-vote-responsiveness'. 'Monotonicity' states that, if some votes change in a certain direction (for example, from 'against' to 'for' a proposition) while all other votes remain fixed, then the social decision should not change in the opposite direction. 'One-vote-responsiveness' states that, starting from a situation in which the decision is one of social indifference, the change of one vote in a certain direction should be enough to break the social indifference in the direction of the change (for example, if one person who initially opposes a proposition changes to vote in favour of it, then the social decision should also change to favour the proposition). ${ }^{26}$

'Monotonicity' and 'one-vote-responsiveness' capture some important democratic desiderata associated with Simple-Majority rule. 'Monotonicity' requires social decisions to be a positive (precisely: non-negative) function of how people vote, which is the essence of democracy. 'One-vote-responsiveness' captures the idea that every single vote counts, by ensuring that in the case of a tie the change of a single vote determines the outcome.

May's Theorem states that Simple-Majority rule is the unique voting rule that satisfies all of May's conditions simultaneously. All other voting rules violate at least one condition.

All voting rules of a 'majoritarian type' considered here - simple and special ones alike - satisfy 'universal domain', 'anomyity' and 'monotonicity' (see Appendix I).

Asymmetrical Special-Majority rules violate May's 'symmetry' condition, while Symmetrical Special-Majority rules satisfy that condition. To justify an Asymmetrical Special-Majority rule, therefore, we need some justification for the asymmetry (for the 'bias' in favour of the default option) - and also for the 'size' of that asymmetry (as reflected in the size of the special majority required for the other option to prevail).

By contrast, Symmetrical Special-Majority rules violate 'one-vote-responsiveness' (they are responsive only to a change of enough votes to constitute a 'special majority'), while Asymmetrical Special-Majority rules satisfy that condition. Of course, whatever reasons we have for requiring a 'special majority' to make a decision, those might also constitute reasons for modifying the responsiveness requirement accordingly. Below we generalize the condition of 'one-vote-responsiveness' to that of ' $k$-votes-responsiveness', where $k$ is the number of votes sufficient to break a tie. May's condition corresponds to

${ }^{24}$ Beitz, Political Equality, p. 59.

25 Bruce Ackerman, Social Justice in the Liberal State (New Haven, Conn.: Yale University Press, 1980), p. 279, see also pp. 11-12, 44-5, 277-85. In 1951, 'The Case for Bare Majority Rule', Ethics, 62 (1951), 6-32, at p. 17, Neil Reimer makes a similar point when complaining that (implicitly, Asymmetrical) special-majority rules violate the 'egalitarian premise' central to democratic rule 'that each citizen ... has the right to have his vote for elected officials counted equally with others'. Under an (Asymmetrical) special-majority rule, 'the views of the individual members of the minority would be more heavily weighted than those of the individuals composing the majority'.

${ }^{26}$ May's original condition of 'positive responsiveness' is essentially the conjunction of these two conditions; it states that, starting from a situation in which the decision is either one of social indifference or one of acceptance of a proposition, if one vote changes in the direction of that proposition (i.e. away from opposing it or towards supporting it, which is equivalent under our simplifying assumption that individual voters are not indifferent), then the decision should become or remain one of acceptance of the proposition. 
the special case of $k=1$. While violating 'one-vote-responsiveness', Symmetrical Special-Majority rules satisfy the less demanding condition of ' $k$-votes-responsiveness' for a suitable $k$. This modification comes at the price of a proliferation of what we call 'non-trivial ties'. Where no option receives the requisite 'special majority', a Symmetrical Special-Majority rule deems the decision to be a 'tie'; and such ties may occur even if one option receives more votes than the other (just insufficiently many more). ${ }^{27}$

Below, we identify a 'trilemma'. If we want to give all minorities above a certain size a 'veto power' (which is what special-majority rules do), then we must sacrifice either the condition of 'symmetry' or that of 'no non-trivial ties'. ${ }^{28}$ We prove that a voting rule can satisfy any two of those conditions - veto powers; symmetry; no non-trivial ties - but no voting rule can satisfy all three. Simple-Majority rule satisfies the last two but forsakes the first (it allows no vetoes). Asymmetrical Special-Majority rules satisfy the first and last but forsake the middle (they lack symmetry). Symmetrical Special-Majority rules satisfy the first and second but forsake the last (they allow non-trivial ties).

\section{A Formal Statement: The Framework ${ }^{29}$}

We suppose that $n$ individuals have to make a collective decision over two options, for example, the acceptance or rejection of some proposition, or two alternatives or candidates in an election. The individuals are labelled $1,2, \ldots, n$, the options are labelled 1 and -1 .

The vote of individual $i$ is represented by $v_{i}$ (taking the values 1 or -1 ), where $v_{i}=1$ means that individual $i$ votes for option 1 , and $v_{i}=-1$ means that individual $i$ votes for option -1 . For simplicity, we assume that no individual is indifferent between the two options. A profile is a vector $v=\left\langle v_{1}, v_{2}, \ldots, v_{n}\right\rangle$ of votes across the $n$ individuals.

A voting rule is a function $f$ that maps each profile $v$ in a given domain to an outcome $f(v)$ (taking the values 1,0 or -1 ), where:

$f(v)=1$ means that 1 is collectively chosen (a positive decision);

$f(v)=-1$ means that -1 is collectively chosen (a negative decision);

$f(v)=0$ means that 1 and -1 are tied (a tie).

This allows the group to be indifferent between the two alternatives. To define several voting rules formally, let us introduce some notation. Given a profile $v$, we write $\Sigma v_{i}$ as an abbreviation for $v_{1}+v_{2}+\ldots+v_{n}$. Then $\Sigma v_{i}$ is the absolute margin between the number of votes for 1 and the number of votes for -1 , i.e. [number of $1 \mathrm{~s}$ in $v$ ] - [number of $-1 \mathrm{~s}$ in $v$ ]. Now Simple-Majority rule can be defined as follows:

Simple-Majority Rule. For any v,

$$
f(v)=\left\{\begin{array}{rc}
1 & \text { if } \Sigma v_{i}>0 \\
0 & \text { if } \Sigma v_{i}=0 \\
-1 & \text { if } \Sigma v_{i}<0
\end{array}\right.
$$

\footnotetext{
${ }^{27}$ Note that this is not a failure to satisfy the 'universal domain'. A 'tie' is still a decision. No profiles of votes are deemed inadmissible; for some profiles of votes, the two options simply stand in a relation of indifference to each another.

${ }^{28}$ We are here concerned with giving veto power to minorities generically, simply by virtue of their being a minority, rather than giving veto power to specific minority groups. Giving veto power to specific, identified groups would involve a relaxation of the 'anonymity' condition. Giving veto power to minorities, generically, however, is consistent with 'anonymity', but requires the relaxation of either 'symmetry' or 'no non-trivial ties', as discussed here.

${ }^{29}$ Proofs of the results in Section I are given in Appendix II.
} 
Examples of less attractive voting rules are the following:

Dictatorship. For any $v, f(v)=v_{i}$, where $i$ is some antecedently fixed individual.

Imposed Acceptance. For any $v, f(v)=1$.

Imposed Rejection. For any $v, f(v)=-1$.

Imposed Indifference. For any $v, f(v)=0$.

A procedural argument for a particular voting rule is an argument that this rule has certain desirable procedural properties.

\section{The Properties of Simple-Majority Rule}

May's Theorem states that Simple-Majority rule is the unique voting rule that satisfies the following conditions:

Universal domain $(U)$. The domain of $f$ is the set of all logically possible profiles.

Anonymity (A). For any two profiles $v$ and $w$, if $v$ and $w$ are permutations of each other, then $f(v)=f(w)$.

$\operatorname{Symmetry}(S)$. For any profile $v, f(-v)=-f(v)$.

We write $v \geq w$ if, for every $i, v_{i} \geq w_{i}$. We write $v>w$ if $v \geq w$ and not $v=w$.

Monotonicity (M). For any two profiles $v$ and $w, v \geq w$ implies $f(v) \geq f(w)$.

One-vote responsiveness $\left(V R_{l}\right)$. For any two profiles $v$ and $w$, if $f(w)=0$ and $v>w$, then $f(v)=1$.

Theorem 1 (May's Theorem). A voting rule satisfies (U), (A), (S), (M) and $\left(\mathrm{VR}_{1}\right)$ if and only if it is Simple-Majority rule.

To the extent that the conditions of May's Theorem are desirable procedural properties, May's Theorem provides a procedural argument for Simple-Majority rule. Let us briefly consider the properties of Simple-Majority Voting.

(1) Symmetry. As noted above, Simple-Majority rule satisfies condition (S). Swapping all votes for 1 and -1 implies that the collective choice is swapped correspondingly.

(2) Responsiveness, Ties and Tie-breaking. As noted above, Simple-Majority rule satisfies condition $\left(\mathrm{VR}_{1}\right)$. So Simple-Majority rule is very responsive to individual votes in the sense that, given a tie between the two options, the change of even a single vote will break the tie in the direction of that change. As a result, Simple-Majority rule generates very few ties. A voting rule $f$ generates a tie for the profile $v$ if $f(v)=0$. If a tie occurs where the number of votes for 1 equals that for -1 , i.e. $f(v)=0$ and $\Sigma v_{i}=0$, we say that the tie is trivial. If a tie occurs although one option receives more votes than the other, i.e. $f(v)=0$ and $\Sigma v_{i} \neq 0$, we say that the tie is non-trivial. Simple-Majority Voting does not generate any non-trivial ties:

No non-trivial ties (NT). For any profile $v, f(v)=0$ implies $\Sigma v_{i}=0$.

In fact, we can characterize Simple-Majority rule by replacing condition $\left(\mathrm{VR}_{1}\right)$ in May's Theorem with condition (NT).

Corollary of May's Theorem. A voting rule satisfies (U), (A), (S), (M) and (NT) if and only if it is Simple-Majority rule. 
(3) Veto Powers. Under Simple-Majority rule, a group of $n / 2$ or more of the individuals can veto a positive decision; and a group of $n / 2$ or more of the individuals can also veto a negative decision. Formally, consider the following two conditions:

Veto over positive decisions for a group of size $k\left(P V_{k}\right)$. For any profile $v$, if there are at least $k$ individuals $i$ such that $v_{i}=-1$, then $f(v) \neq 1$.

Veto over negative decisions for a group of size $k\left(N V_{k}\right)$. For any profile $v$, if there are at least $k$ individuals $i$ such that $v_{i}=1$, then $f(v) \neq-1$.

Simple-Majority rule satisfies both $\left(\mathrm{PV}_{k}\right)$ and $\left(\mathrm{NV}_{k}\right)$ with $k=n / 2$. But it does not satisfy either condition for any integer $k<n / 2$. So no minorities - groups of size less than $n / 2$ - have any veto powers under Simple-Majority Voting.

\section{The Trilemma between Symmetry, No Non-trivial Ties and Minority Veto Powers}

We have seen that Simple-Majority rule satisfies symmetry and no non-trivial ties, but it does not give any veto powers to minorities. Are there any other voting rules satisfying all of

(1) symmetry,

(2) no non-trivial ties,

(3) giving certain veto powers to minorities?

Theorem 2 (procedural trilemma). For any integer $k<n / 2$, there exists no voting rule satisfying (U), (S), (NT) and $\left(\mathrm{PV}_{k}\right)\left(\right.$ or $\left.\left(\mathrm{NV}_{k}\right)\right)$.

We are faced with a trilemma. No voting rule can satisfy all three of (1), (2) and (3), but any two of (1), (2) and (3) can be simultaneously satisfied. Simple-Majority rule satisfies (1) and (2) while violating (3). In fact, we have:

Proposition 1. A voting rule satisfies (U), (NT), (S) and $\left(\mathrm{PV}_{n / 2}\right)$ (and $\left(\mathrm{NV}_{n / 2}\right)$ ) if and only if it is Simple-Majority rule.

If we want to ensure certain minority veto powers, we need to relax either (1) or (2). Asymmetrical Special-Majority rules satisfy (2) and (3) while violating (1). Symmetrical Special-Majority rules satisfy (1) and (3) while violating (2).

\section{Asymmetrical Special-Majority Rules}

If we relax symmetry but do not permit non-trivial ties, not only is one alternative always privileged over the other; the minority veto powers the special-majority rule grants are then themselves also asymmetrical. There is always, in that case, a trade-off between minority veto powers over negative decisions and minority veto powers over positive decisions.

Proposition 2. If a voting rule satisfies (U), (NT), $\left(\mathrm{PV}_{k_{1}}\right)$ and $\left(\mathrm{NV}_{k_{2}}\right)$, then $k_{1}+k_{2} \geq n$.

If we give a minority of size $k_{1}<n / 2$ veto power over positive decisions, then at most a supermajority of size greater than $n-k_{1}>n / 2$ has veto power over negative 
decisions, and vice versa. An Asymmetrical Special-Majority rule can be defined as follows:

Asymmetrical Special-Majority Rule with parameter $m$. For any $v$,

$$
f(v)=\left\{\begin{array}{rl}
1 & \text { if } \sum v_{i} \geq m \\
-1 & \text { if } \sum v_{i}<m
\end{array} \quad(m>n \text { or } m<-n \text { is admissible }) .\right.
$$

If $m>0$ (if $n$ is even) or $m>1$ (if $n$ is odd), the Asymmetrical Special-Majority rule is biased in favour of -1 . In that case, a minority of size greater than $(n-m) / 2$ can veto a positive decision; but only a supermajority of size at least $(n+m) / 2$ can veto a negative decision. If $m \leq 0$ (if $n$ is even) or $m \leq-1$ (if $n$ is odd), the rule is biased in favour of 1 . In that case, any minority of size greater than $(n+m-1) / 2$ can veto a negative decision; but only a supermajority of size at least $(n-m+1) / 2$ can veto a positive decision. ${ }^{30}$

Proposition 3. An Asymmetrical Special-Majority rule with parameter $m$ satisfies $\left(\mathrm{PV}_{k}\right)$ if and only if $k>(n-m) / 2$, and it satisfies $\left(\mathrm{NV}_{k}\right)$ if and only if $k>(n+m-1) / 2$.

\section{Symmetrical Special-Majority Rules}

If we keep symmetry, but permit non-trivial ties, then it is the case not only that no alternative is privileged over the other, but also that the minority veto powers that the special majority rule grants are always symmetrical.

Proposition 4. Suppose a voting rule satisfies (U) and (S). Then, for any $k$, it satisfies $\left(\mathrm{PV}_{k}\right)$ if and only if it satisfies $\left(\mathrm{NV}_{k}\right)$.

A Symmetrical Special-Majority rule can be defined as follows:

Symmetrical Special-Majority Rule with parameter $m(m>0)$. For any $v$,

$$
f(v)=\left\{\begin{aligned}
1 & \text { if } \Sigma v_{i} \geq m \\
0 & \text { if } m>\sum v_{i}>-m \\
-1 & \text { if } \Sigma v_{i} \leq-m \quad(m>n \text { is admissible }) .
\end{aligned}\right.
$$

The limiting case $m=1$ corresponds to Simple-Majority rule. The condition $\Sigma v_{i} \geq m$ means that there is a special majority for 1 with a margin of at least $m$ between the majority and the minority. The condition $\Sigma v_{i} \leq-m$ means that there is a special majority for -1 with a margin of at least $m$ between the majority and the minority. The condition $m>\Sigma v_{i}>-m$ means that there is no sufficient special majority for either 1 or -1 .

The class of symmetrical special majority rules can be fully characterized by May's conditions (U), (A), (S), (M), where condition $\left(\mathrm{VR}_{1}\right)$ is relaxed.

Theorem 3. A voting rule satisfies (U), (A), (S) and (M) if and only if it is a Symmetrical Special-Majority rule for some parameter $m>0$.

Theorem 3 characterizes a whole class of voting rules. This class includes, for example, Simple-Majority rule $(m=1)$, the Unanimity Rule $(m=n)$, the Imposed Indifference rule $(m>n)$. For a suitable choice of $m>1$, minorities have veto powers over both positive and negative decisions (recall Proposition 4 above).

${ }^{30}$ To make the special majority more demanding than a simple majority, we need to require [ $m>1$ or $\left.m \leq-1\right]$ if $n$ is odd, and [ $m>2$ or $m \leq-2$ ] if $n$ is even. 
To characterize not just the class of all Symmetrical Special-Majority rules, but specific such rules, we can use a minority veto condition to impose a lower bound on $m$, and a generalized responsiveness condition to impose an upper bound on $m$. A lower bound on $m$ can be obtained as follows.

Proposition 5. A Symmetrical Special-Majority rule with parameter $m$ satisfies $\left(\mathrm{PV}_{k}\right)$ (and hence $\left(\mathrm{NV}_{k}\right)$ ) if and only if $n-2 k<m$.

To obtain an upper bound on $m$, we generalize the condition of one-vote responsiveness introduced above (May's condition is the special case for $k=1$ ).

$k$-Votes Responsiveness $\left(V R_{k}\right)$. For any two profiles $v$ and $w$, if $f(w)=0, v \geq w$, and there are at least $k$ individuals $i$ such that $v_{i}>w_{i}$, then $f(v)=1$.

A voting rule satisfies $k$-votes responsiveness if, in the case of a tie, the change of $k$ votes (all in the same direction, specifically from -1 to 1 ) will break the tie in the direction of that change (also from -1 to 1 ). Now an upper bound on $m$ can be obtained as follows.

Proposition 6. Let $m$ be any integer greater than 0 (where $m$ is even if $n$ is even, and odd if $n$ is odd). A Symmetrical Special-Majority rule with parameter $m$ satisfies $\left(\mathrm{VR}_{k}\right)$ if and only if $m<k+2$.

Now May's conditions (U), (A), (S), (M) together with Propositions 5 and 6 allow us to characterize Symmetrical Special-Majority rules for a specific range of parameters $m$.

Proposition 7. Let $m$ be any integer greater than 0 (where $m$ is even if $n$ is even, and odd if $n$ is odd). A voting rule satisfies (U), (A), (S), (M), $\left(\mathrm{PV}_{k_{1}}\right)$ and $\left(\mathrm{VR}_{k_{2}}\right)$ if and only if it is a Symmetrical Special-Majority rule with parameter $m$ where $n-2 k_{1}<m<k_{2}+2$.

If more than one value of $m$ (where $m$ is even if $n$ is even, and odd if $n$ is odd) satisfies $n-2 k_{1}<m<k_{2}+2$, the conditions of Proposition 7 characterize a range of Symmetrical Special-Majority rules. If exactly one value of $m$ satisfies the inequality, the conditions characterize a specific Symmetrical Special-Majority rule uniquely. If no value of $m$ satisfies the inequality - i.e. if $k_{2} \leq n-2 k_{1}$ - then the conditions of Proposition 7 cannot be satisfied, i.e. we have an impossibility result. So the trade-off between minority veto powers and responsiveness under Symmetrical Special-Majority Voting is as follows:

Proposition 8. If a Symmetrical Special-Majority rule satisfies both $\left(\mathrm{PV}_{k_{1}}\right)$ and $\left(\mathrm{VR}_{k_{2}}\right)$, then $k_{2}>n-2 k_{1}$.

The more responsive the voting rule (i.e. the smaller the value of $k_{2}$ in ' $k_{2}$-votes responsiveness'), the larger the group size $k_{1}$ that is required for vetoing a (positive or negative) decision. Condition $\left(\mathrm{VR}_{1}\right)\left(\right.$ where $\left.k_{2}=1\right)$, as satisfied by Simple-Majority rule, implies $1=k_{2}>n-2 k_{1}$, i.e. $k_{1}>n / 2-1 / 2$, and thus rules out minority veto powers.

\section{EPISTEMIC PROPERTIES OF SIMPLE AND SPECIAL-MAJORITY RULES}

Democratic procedures commend themselves not only on the grounds of procedural fairness, such as those formalized in May's Theorem. They also commend themselves on epistemic grounds, in terms of their truth-tracking power. 
Aristotle's loose talk of the 'wisdom of the multitude' was formalized in the Condorcet Jury Theorem in the eighteenth century and has been intensively explored in recent years. ${ }^{31}$ The theorem shows that, if individuals cast their votes independently of one another and each voter is more than 0.5 likely to be correct in a two-option choice, the probability that the majority vote is correct is an increasing function of the size of the electorate, approaching certainty as the number of individuals tends to infinity. Majority voting is, in that sense, a good truth-tracker.

\section{An Informal Statement}

Here we explore a Bayesian version of the familiar Condorcet Jury model, to reveal an epistemic trilemma analogous to the procedural one above. ${ }^{32}$ The role of a 'minority veto' condition in the procedural case is taken by a 'no reasonable doubt' condition in the epistemic one. The issues discussed here arise in various circumstances, legal, medical and administrative. ${ }^{33}$

Sometimes we want to make very certain we are right before acting. Members of a criminal jury are asked to convict only if they are convinced 'beyond a reasonable doubt' of the defendant's guilt: something like a 95 per cent probability that the defendant is guilty. In civil trials, in contrast, the standard of proof is merely 'more likely than not': a probability just over 50 per cent, either way, is sufficient for a decision.

Sometimes we think that the evidentiary burden ought to weigh disproportionately in one direction. In the criminal jury case, while the prosecution has to prove its case beyond a reasonable doubt, the defence does not. Other times, we think that the evidentiary burden ought to be symmetrical, as in civil cases.

Sometimes, yet again, we think that the standard of proof should be 'no reasonable doubt', but that that standard should apply symmetrically to both sides of the proposition. Suppose, for example, we are dealing with a drug that would, at worst, have only mildly unpleasant side-effects; and that, at best, would alleviate a condition which is only mildly unpleasant. There we might suppose: (1) the state should allow the sale of the drug under the imprimatur of a 'licensed and approved therapeutic agent' only upon production of evidence that it is 90 per cent certain that the drug is safe and effective in alleviating the condition; (2) the state should prohibit the sale of the drug only if it is 90 per cent certain that it does more harm than good; and (3) the state should allow the drug to be sold over the counter as a 'folk remedy', but without any official imprimatur, if neither of those conditions is met.

The form that the trilemma takes in the epistemic case is this. There are three properties we might like to see in our epistemic decision procedure. One is 'symmetry' in the epistemic sense: positive decisions are held to the same standard of proof as negative ones. A second is an epistemic equivalent of 'no non-trivial ties' (ties occur only where the probability of the truth of a proposition equals that of its negation). The third is a "no

31 Bernard Grofman, Guillermo Owen and Scott L. Feld, 'Thirteen Theorems in Search of the Truth', Theory and Decision, 15 (1983), 261-78; Mueller, Public Choice III, pp. 128-33.

32 Analogous results are restated in Appendix IV using statistical models of hypothesis testing of a non-Bayesian sort, so the argument can be developed without referring to Bayesian prior probabilities at all.

${ }^{33}$ See, respectively: John Kaplan, 'Decision Theory and the Fact-finding Process', Stanford Law Review, 20 (1968), 1065-92; and Kaplow, 'The Value of Accuracy in Adjudication'; Thomas J. Scheff, 'Decision Rules, Types of Error and their Consequences in Medical Diagnosis', Behavioral Science, 8 (1964), 97-107; and Robert E. Goodin, 'Erring on the Side of Kindness in Social Welfare Policy', Policy Sciences, 18 (1985), 141-56. 
reasonable doubt' standard, requiring more than a 'more-likely-than-not' threshold to be crossed before we decide for or against some proposition.

The trilemma, epistemically, is that any two of those conditions can be met - but not all three at once. Assuming independent voters each of whom is more likely to be right than wrong, Simple-Majority rule meets the first and second conditions but not the third. Suitable Asymmetrical Special-Majority rules meet the second and third but not the first. Suitable Symmetrical Special-Majority rules meet the first and third but not the second.

Here again, we sometimes have grounds for sacrificing one of those conditions. Which voting rule we want to adopt, on epistemic grounds, follows from those reasons we have for considering one or another condition more important, in any given situation. ${ }^{34}$

\section{A Formal Statement: The Framework ${ }^{35}$}

We begin by stating Condorcet's classical model of jury decisions. We assume that there are two possible states of the world, represented by the variable $X$, which takes the value 1 or -1 . The two possible states of the world might be, respectively, the guilt or innocence of a defendant, or the truth or falsity of some factual proposition. Again, we assume that there are $n$ individuals, labelled $1,2, \ldots, n$. The individuals are treated as diagnostic devices whose votes are signals about the state of the world. The process by which each individual $i$ generates his or her vote is represented by the random variable $V_{i}$, where $V_{i}$ takes the value 1 or -1 . Let $V$ denote the vector $\left\langle V_{1}, V_{2}, \ldots, V_{n}>\right.$ of such random variables across the $n$ individuals. For each individual $i$, a specific value of $V_{i}-$ i.e. a specific vote of that individual - is represented by $v_{i}$. As before, a profile is a vector $v=\left\langle v_{1}, v_{2}, \ldots, v_{n}\right\rangle$ of specific such votes. Condorcet's model makes two assumptions, which we will tentatively retain throughout the following discussion and results. ${ }^{36}$

34 As we have pointed out, the drawback of Symmetrical Special-Majority Voting, epistemically as well as procedurally, is that it allows non-trivial ties. But in the epistemic case, non-trivial ties turn out to be less of a problem. In a sufficiently large electorate, the probability of non-trivial ties under Symmetrical Special-Majority Voting can be proved to be vanishingly small. This follows from the fact that Symmetrical Special-Majority Voting, as defined here (in terms of a required absolute margin of votes between the majority and the minority) satisfies the condition of truth-tracking in the limit: the probability of obtaining a special majority for 1 if $X=1$ converges to 1 as $n$ increases; likewise, the probability of obtaining a special majority for -1 if $X=-1$ converges to 1 as $n$ increases. See List, 'On the Significance of the Absolute Margin', and 'The Epistemology of Special Majority Voting'.

${ }^{35}$ Proofs of the results in Section II are given in Appendix III.

36 Many important modifications of Condorcet's model have been discussed in the literature. Cases where different jurors have different competence levels - i.e. where the present homogeneous competence assumption does not hold - are discussed in Grofman, Owen and Feld, 'Thirteen Theorems in Search of the Truth'; P. J. Borland, 'Majority Systems and the Condorcet Jury Theorem', Statistician, 38 (1989), 181-9; S. Kanazawa, 'A Brief Note on a Further Refinement of the Condorcet Jury Theorem for Heterogeneous Groups', Mathematical Social Sciences, 35 (1998), 69-73. Cases where there are certain dependencies between different jurors' votes - i.e. where the present independence assumption does not hold - are discussed in Krishna Ladha, 'The Condorcet Jury Theorem, Free Speech and Correlated Votes', American Journal of Political Science, 36 (1992), 617-34; David Estlund, 'Opinion Leaders, Independence and Condorcet's Jury Theorem', Theory and Decision, 36 (1994), 131-62; and Franz Dietrich and Christian List, 'A Model of Jury Decisions Where All Jurors Have the Same Evidence', Synthese, 142 (2004), 175-202. Cases where jurors vote strategically rather than sincerely - i.e. where jurors may not vote their private signals about the state of the world - are discussed in David Austen-Smith and Jeff S. Banks, 'Information Aggregation, Rationality and the Condorcet Jury Theorem', American Political Science Review, 90 (1996), 34-45. But, for the purposes of this article, we tentatively use Condorcet's model in its simplest, classical form. 
First, if the state of the world is 1 , the individuals each have a greater than $1 / 2$ chance of voting for 1 ; and if it is -1 , they each have a greater than $1 / 2$ chance of voting for -1 .

Competence. For each individual $i, \operatorname{Pr}\left(V_{i}=1 \mid X=1\right)=\operatorname{Pr}\left(V_{i}=-1 \mid X=-1\right)=p>1 / 2$, where $p$ (the individual competence level) is the same for all individuals.

The probability $\operatorname{Pr}\left(V_{i}=1 \mid X=1\right)$ (respectively $\operatorname{Pr}\left(V_{i}=-1 \mid X=-1\right)$ ) is the conditional probability that individual $i$ votes for 1 (respectively -1 ), given that the state of the world is 1 (respectively -1 ).

Secondly, once the state of the world is given, the votes of different individuals are independent from each other.

Independence. The votes of different individuals $V_{1}, V_{2}, \ldots, V_{n}$ are independent, given the state of the world $X$.

In short, the votes of different individuals are independent identically distributed signals about the state of the world, where each signal is noisy but biased towards the truth.

The key idea of an epistemic account of voting is that a particular voting pattern provides evidence about the state of the world, and that a good evaluation of that evidence - using a suitable voting rule - allows a group to make decisions that track the state of the world reliably. An epistemic argument for a particular voting rule is an argument that a group using this voting rule will be good at making decisions that track the state of the world reliably.

\section{The Properties of Simple-Majority Rule}

Let us first address the properties of Simple-Majority rule from an epistemic perspective.

(1) The standard of proof. Suppose we assign an equal prior probability of $1 / 2$ to each of the two states of the world, 1 and -1 . This need not be an objective probability; in the absence of more precise information, we might justify this equiprobability assumption by some normative principle ('no bias') or some methodological principle (Laplace's 'principle of insufficient reason'). Condorcet's own presentation implicitly relied on this assumption. ${ }^{37}$ While the present exposition uses Bayesian notions and therefore requires a prior probability assignment over the different states of the world, we present a classical (non-Bayesian) statistical variant of the present results in Appendix IV, which requires no assumption about prior probabilities at all.

The first thing to note is that, other things being equal, observing an individual vote for 1 (respectively -1 ) should increase our degree of belief in the hypothesis that the state of the world is 1 (respectively -1 ). Observing more such votes should increase our degree of belief in that hypothesis further. Whenever we observe a majority of votes for 1 , this should lead us to believe that $X=1$ is more likely to be true than $X=-1$. Likewise, whenever we observe a majority for -1 , this should lead us to believe that $X=-1$ is more likely to be true than $X=1$. In short, under Simple-Majority Voting, a positive decision is made if and only if $X=1$ is more likely to be true than $X=-1$; a negative decision is made if and only if $X=-1$ is more likely to be true than $X=1$.

${ }^{37}$ List, 'On the Significance of the Absolute Margin'. 
However, in many situations, we require that a positive decision be made, not as soon as $X=1$ is more likely to be true than $X=-1$, but only if we believe, beyond any reasonable doubt, that $X=1$ is true. Consider the following two conditions:

A standard of proof of c for positive decisions $\left(P P_{c}\right)$. For any profile $v, f(v)=1$ if and only if $\operatorname{Pr}(X=1 \mid V=v)>c$.

A standard of proof of $c$ for negative decisions $\left(N P_{c}\right)$. For any profile $v, f(v)=-1$ if and only if $\operatorname{Pr}(X=-1 \mid V=v)>c$.

The probability $\operatorname{Pr}(X=1 \mid V=v)$ (respectively $\operatorname{Pr}(X=-1 \mid V=v)$ ) is the conditional probability that the state of the world is 1 (respectively -1 ), given that the pattern of votes across the $n$ individuals is precisely the profile $v$. The parameter $c$ captures the requisite standard of proof. The conditions require that a positive (respectively negative) decision be made if and only if the conditional probability that $X=1$ (respectively $X=-1$ ), given the voting pattern, exceeds the threshold $c$.

As we have noted, Simple-Majority rule satisfies $\left(\mathrm{PP}_{c}\right)$ and $\left(\mathrm{NP}_{c}\right)$ for $c=1 / 2$. But Simple-Majority rule does not satisfy either $\left(\mathrm{PP}_{c}\right)$ and $\left(\mathrm{NP}_{c}\right)$ for any value of $c$ significantly greater than $1 / 2$. We say, in a technical sense, that $c$ is significantly greater than $1 / 2$ if

$$
c \geq \begin{cases}p & \text { if } n \text { is odd } \\ \left.p^{2} /\left(p^{2}+(1-p)^{2}\right)\right) & \text { if } n \text { is even. }\end{cases}
$$

Intuitively, only a value of $c$ close enough to 1 and thus typically significantly greater than $1 / 2$ - say $c=0.95$ - will capture the requirement of 'no reasonable doubt'. So Simple-Majority Voting is an unsuitable voting rule if we demand a threshold of "no reasonable doubt' that is significantly greater than $1 / 2$.

(2) Symmetry. In the procedural case, we defined symmetry as the requirement that swapping all votes for 1 and -1 implies that the outcome of the aggregation is swapped correspondingly. But symmetry can also be defined in epistemic terms, namely as the requirement that the standard of proof for positive decisions should be exactly the same as that for negative decisions; in other words, that a voting rule should satisfy $\left(\mathrm{PP}_{c}\right)$ for some value of $c$ if and only if it satisfies $\left(\mathrm{NP}_{c}\right)$ for the same value of $c$. Under Condorcet's assumptions - including, crucially, the assignment of an equal prior probability to the two states of the world ${ }^{38}$ - Simple-Majority Voting satisfies symmetry in this sense.

(3) Ties. We have seen that Simple-Majority Voting rules out non-trivial ties in a procedural sense: it allows ties only when the number of individuals voting for 1 equals the number of individuals voting for -1 . There is also an epistemic sense in which Simple-Majority Voting rules out non-trivial ties. If $f(v)=0$ and $\operatorname{Pr}(X=1 \mid V=v)=1 / 2$, we say that the tie is trivial. In that case, the tie occurs in a situation where we consider the two possible states of the world equally probable. If $f(v)=0$ and $\operatorname{Pr}(X=1 \mid V=v) \neq 1 /$ 2 , by contrast, we say that the tie is non-trivial. In that case, there is a tie although we consider one of the two possible states of the world

\footnotetext{
38 Again, the assumption is not needed in the classical (non-Bayesian) version of the argument in Appendix IV.
} 
more probable than the other. Simple-Majority Voting does not allow any non-trivial ties in this epistemic sense. It satisfies: ${ }^{39}$

No non-trivial ties (NT*). For any profile $v, f(v)=0$ implies $\operatorname{Pr}(X=1 \mid V=v)=$ $\operatorname{Pr}(X=-1 \mid V=v)=1 / 2$.

\section{The Trilemma Between Symmetry, No Non-trivial Ties and No Reasonable Doubt}

We have seen that Simple-Majority Voting satisfies both symmetry and no non-trivial ties in the epistemic sense, but it cannot implement a threshold of 'no reasonable doubt' significantly greater than $1 / 2$ for either positive or negative decisions. In analogy with the procedural case, we may ask whether there are any other voting rules satisfying all the following:

(1) symmetry in the epistemic sense,

(2) no non-trivial ties in the epistemic sense,

(3) no reasonable doubt.

The following result gives a negative answer to this question.

Theorem 4. For any standard of proof parameter $c$ significantly greater than $1 / 2$, there exists no voting rule satisfying $(\mathrm{U}),\left(\mathrm{NT}^{*}\right),\left(\mathrm{PP}_{c}\right)$ and $\left(\mathrm{NP}_{c}\right)$.

Again, we are faced with a trilemma. No voting rule can satisfy all three of (1), (2) and (3), but any two of (1), (2) and (3) are satisfiable. Simple-Majority Voting satisfies (1) and (2) while violating (3). In fact, Simple-Majority Voting is the unique voting rule satisfying $\left(\mathrm{PP}_{1 / 2}\right)$ and $\left(\mathrm{NP}_{1 / 2}\right)$ together with universal domain:

Proposition 9. In Condorcet's model, a voting rule satisfies $(\mathrm{U}),\left(\mathrm{PP}_{1 / 2}\right)$ and $\left(\mathrm{NP}_{1 / 2}\right)$ if and only if it is Simple-Majority Voting.

If we want to ensure a standard of proof significantly greater than $1 / 2-$ e.g. a threshold of 'no reasonable doubt' - we need to relax either (1) or (2). An Asymmetrical Special-Majority rule satisfies (2) and (3) while violating (1). A Symmetrical SpecialMajority rule satisfies (1) and (3) while violating (2).

Asymmetrical Special-Majority rules. If we relax symmetry but do not permit non-trivial ties, we are faced with a trade-off between standards of proof for positive and negative decisions.

Proposition 10. Suppose a voting rule satisfies (U) and (NT*), and suppose $c_{1}$ is significantly greater than $1 / 2$. If the voting rule satisfies $\left(\mathrm{PP}_{c_{1}}\right)$, then it does not satisfy $\left(\mathrm{NP}_{c_{2}}\right)$ for any $c_{2} \geq 1 / 2$; and if it satisfies $\left(\mathrm{NP}_{c_{1}}\right)$, then it does not satisfy $\left(\mathrm{PP}_{c_{2}}\right)$ for any $c_{2} \geq 1 / 2$.

If we demand a standard of proof for positive decisions that is significantly greater than $1 / 2$, then we cannot also demand a standard of proof for negative decisions that is greater than or equal to $1 / 2$. Likewise, if we demand a standard of proof for negative decisions that is significantly greater than $1 / 2$, then we cannot also demand a standard of proof for negative decisions that is greater than or equal to $1 / 2$.

39 Assuming an equal prior probability of the two states of the world - this assumption is not needed in the classical (non-Bayesian) version of the argument in Appendix IV. 
In jury decisions this seems acceptable, as the standard of proof for conviction should be higher than that for acquittal. But in other decision problems, where there is no antecedently privileged alternative, we may require a symmetrical standard of proof. And if we require not only a standard of proof that is symmetrical, but also one that is significantly greater than $1 / 2$, then we are led to a Symmetrical Special-Majority rule. $^{40}$

\section{Symmetrical Special-Majority Rules}

We can now provide a characterization result on Symmetrical Special-Majority rules. If we permit non-trivial ties in the epistemic sense, then Symmetrical Special-Majority rules are the unique voting rules satisfying universal domain and a symmetrical standard of proof. When the required standard of proof $c$ and the individual competence parameter of $p$ are given, the parameter $m$ of the corresponding Symmetrical Special-Majority rule can be determined by the expression $\log \left({ }^{1} / c-1\right) / \log \left({ }^{1} / p-1\right)$.

Proposition 11. Let $c \geq 1 / 2$. A voting rule satisfies $(\mathrm{U}),\left(\mathrm{PP}_{c}\right)$ and $\left(\mathrm{NP}_{c}\right)$ if and only if it is a Symmetrical Special-Majority rule, where the parameter $m$ is the smallest integer strictly greater than $\log (1 / c-1) / \log (1 / p-1)$.

The case $c=1 / 2$ corresponds to Simple-Majority rule. The case $c$ significantly greater than $1 / 2$ but less than $\left.p^{n} /\left(p^{n}+(1-p)^{n}\right)\right)$ corresponds to a Special-Majority (up to Unanimity) rule. The case $c$ greater than or equal to $\left.p^{n} /\left(p^{n}+(1-p)^{n}\right)\right)$ corresponds to Imposed Indifference.

\section{CHOOSING AMONG DECISION RULES}

Figure 1 summarizes the parallel trilemmas identified in Sections I-II. The nodes of the triangle represent three conditions that we might like a voting rule to satisfy, but only two

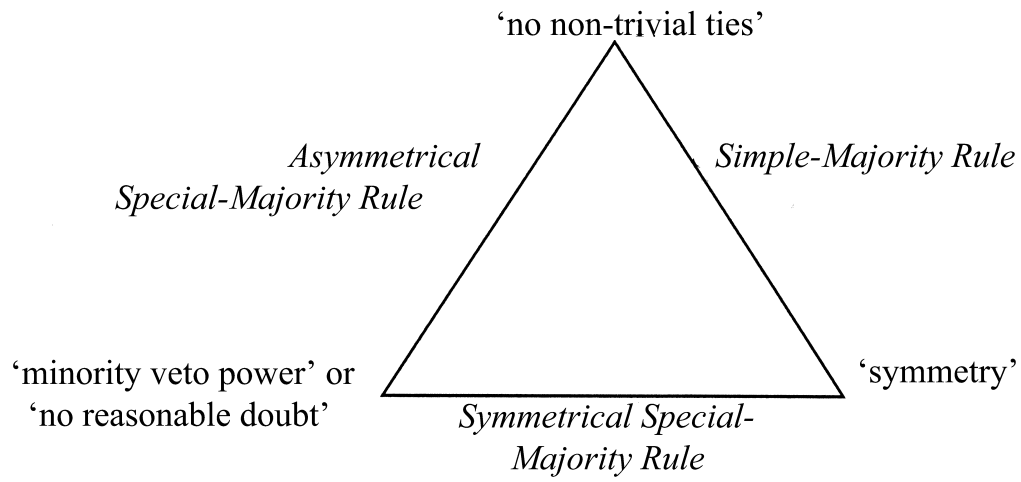

Fig. 1. The trilemmas

40 Once again, it is important to note the assumption of an equal prior probability of both states of the world. If that assumption is given up, then, in a Bayesian framework, a symmetrical standard of proof may lead to (and therefore justify) an asymmetrical Special-Majority rule. In the classical framework in Appendix IV, by contrast, no assumption about prior probabilities is needed to justify a Symmetrical Special-Majority rule on the basis of a symmetrical standard of proof requirement. 
of which can be simultaneously satisfied. Each side of the triangle, connecting two nodes, represents the type of voting rule that satisfies those two conditions (whilst violating the condition at the opposite node). The trilemmas in Figure 1 help us see what is at stake in the choice among alternative voting rules. The decision tree in Figure 2 summarizes that choice.

Procedurally, is there any reason for any subgroups smaller than a majority to get a veto?

Epistemically, is there any reason for demanding a standard of proof higher than 'more likely than not'?

Is there any reason for a

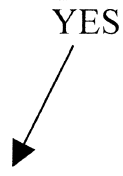

stronger presumption in favour

of one alternative rather than the other?

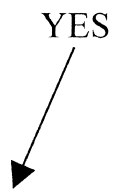

Asymmetrical Special Majority

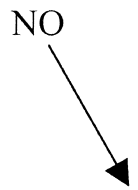

Symmetrical

Special Majority

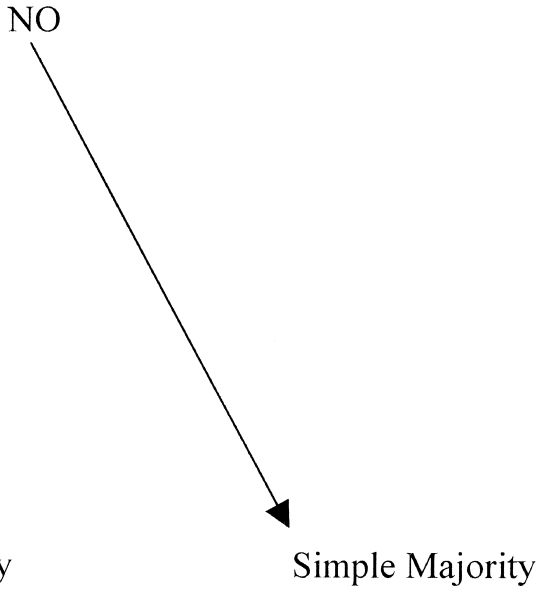

Simple Majority

Fig. 2. Grounds for choosing among decision rules

Procedurally, the great disadvantage of Simple-Majority rule is the risk of majority tyranny. Under Simple-Majority rule, the majority might ride roughshod over the interests of 'discrete and insular' minorities that have distinctive interests but only a minority of the votes. We may wish to protect such minorities by requiring that decisions affecting them be taken by 'special majorities' sufficiently large to, in effect, give such minorities veto power.

Often, of course, there is no such problem. There may be no real risk of any group being so discrete and insular as to be in danger of being tyrannized by a majority. Then Simple-Majority rule is satisfactory. Indeed, in the limiting case where there are absolutely no factions (every pair of voters is as likely to vote with one another as against one another), Simple-Majority rule is the voting rule that uniquely maximizes each voter's probability of being on the winning side of an election. ${ }^{41}$

Thus, Simple-Majority rule works fine where there are no factions or any other reasons to grant submajorities veto power over the social outcome. But where there is a genuine risk of sufficiently cohesive submajorities with sufficiently strong and distinctive interests, we may want to give them extra power over the outcome. Under certain special conditions,

41 Douglas W. Rae, 'Decision-Rules and Individual Values in Constitutional Choice', American Political Science Review, 63 (1969), 40-56; Michael Taylor, 'Proof of a Theorem on Majority Rule', Behavioral Science, 14 (1969), 228-31; Mueller, Public Choice III, pp. 136-7. 
Simple-Majority rule might itself provide them with that (if, for example, the groups in question are pivotal in coalition or majority-cycling situations). ${ }^{42}$ But giving submajorities anything like a strong veto power requires us to abandon Simple-Majority rule in favour of some form of special-majority voting.

Epistemically, likewise, Simple-Majority rule is ideal so long as we merely want to identify propositions that are 'more likely than not' to be true. But if we require greater confidence, we need some form of special-majority voting. ${ }^{43}$

The great disadvantage of ordinary Asymmetrical Special-Majority rules is precisely their asymmetry. They privilege one option as the 'default' one that prevails if the other option does not receive the requisite 'special majority'.

Again, sometimes that is not a problem. There may be good grounds for privileging one option in that way. There are good grounds for a presumption of innocence in criminal trials, and for making it harder to convict than to acquit. There are good grounds for requiring a larger legislative majority to overturn a president's veto than was required to pass the bill in the first place, in order for a mixed constitution to provide genuine checks and balances.

Thus, there exist cases in which the asymmetries built into Asymmetrical SpecialMajority rules are not arbitrary. But the burden must be on advocates of the differential treatment of the various options to provide a justification for the asymmetry.

Symmetrical Special-Majority rules solve that problem by treating all options symmetrically. They require the same 'special majority' for either option in order for it to be chosen. The great disadvantage of a Symmetrical Special-Majority rule is that it may generate many 'non-trivial ties'. It chooses neither option as the social decision if neither achieves the requisite majority - even if one option got more votes than the other.

Sometimes this might not be a problem. Sometimes it does no harm to leave the matter unsettled. But in general, we put something to a vote only when we genuinely need to have the issue resolved; and hence a voting rule that leaves too many things unsettled seems problematic. It is to that problem that we now turn.

\section{BREAKING TIES}

The problem with leaving things formally unsettled is that, as we have long been aware, 'non-decisions' are decisions too. ${ }^{44}$ Something will happen, or not happen, in consequence

\footnotetext{
42 In a spatial voting framework, McCann argues that simple-majority rule protects worst-off minorities better than other (e.g., supermajority) rules. His argument rests on the claim that the instability (cycling) inherent in the simple-majority rule allows minorities to overturn dispreferred outcomes by entering into appropriate coalitions. This is an interesting argument, identifying a potentially desirable implication of the often criticized instability of simple-majority rule. See A. J. McCann, 'The Tyranny of Supermajorities', Journal of Theoretical Politics, 16 (2004), 53-77.

${ }^{43}$ There are also arguments for supermajority rules as a means of inducing greater revelation of information and encouraging the participation of those who might otherwise opt out of the process. See Hao Li, 'A Theory of Conservatism', Journal of Political Economy, 109 (2001), 617-36; and Michael Suk-Young Chwe, 'Minority Voting Rights Can Maximize Majority Welfare', American Political Science Review, 93 (1999), 85-97.

${ }^{4}$ See Peter Bachrach and Morton S. Baratz, 'Decisions and Non-decisions: An Analytic Framework', American Political Science Review, 57 (1963), 632-42. Sunstein uses the phrase to refer to something slightly different: leaving the 'grounds' for a judicial decision open, as judges content themselves with simply rendering a decision in the case at hand (with each judge deciding the case on different grounds, perhaps). See Cass R.Sunstein, 'Leaving Things Undecided', Harvard Law Review, 110 (1996), 4-101.
} 
of things being left undecided; some interests will be well served, and others ill served. ${ }^{45}$ Leaving things undecided is not without consequences. ${ }^{46}$

So it is genuinely a problem that Symmetrical Special-Majority rules may leave things undecided. Notice, however, that most decision rules - including Simple-Majority rule with an even number of voters - have to face the problem of what to do in the case of tied votes. ${ }^{47}$ Ties may occur more frequently under Symmetrical Special-Majority rules, but the problem is in no way unique to them. Examining how that problem is handled in connection with other voting rules gives us some hints as to how we might solve that problem with respect to Symmetrical Special-Majority rules.

Generically, there are three ways of resolving ties. Either: (1) we can privilege one of the options; or (2) we can privilege one of the voters; or (3) we can settle issues on which there are ties by some wholly separate procedure.

Cursory inspection of actual decision procedures reveals many examples of (1). The most familiar is the rule that 'the status quo remains in force unless some alternative to it is enacted'.

There are not many cases of (2). One example rather like that might be the practice of the Speaker of the US House of Representatives casting the deciding vote in cases of a tie. But even that is not a completely clean case of (2). It is not as if the Speaker has a 'golden vote'. Rather (by convention) the Speaker only votes when his vote is 'golden', i.e., in cases of a tie when his vote will indeed decide the matter. ${ }^{48}$

More interesting from our point of view are examples of (3). Note in this connection the procedure set down in the original text of Article II, Section 1 of the US Constitution for choosing the president:

The person having the greatest number of votes [in the Electoral College] shall be the President, if such a number be a majority of the whole number of Electors appointed; and if there be more

45 For a dramatic example, reflect upon this tale from the Ukraine relayed by John Dryzek and Leslie Holmes, Postcommunist Democratization (Cambridge: Cambridge University Press, 2002), p. 117, emphasis added. 'Parliamentary elections were ... held in 1994, contested by a large number of parties, many of which were concentrated in particular regions. The elections were conducted under the requirement that, to be elected, a candidate had to secure the support of over 50 percent of registered voters, either in the first round or in a runoff between the top two candidates in a constituency. The results were hardly conclusive. [Low turnout meant that m] any constituencies could not elect a representative under this system ...' Such non-election inevitably favours one side or another. In the Ukraine, it in effect favoured the Communist Party and the old nomenklatura. They won, not exactly by default, but nonetheless precisely because other seats went unfilled.

${ }^{46}$ In the purple prose of Rae's 'Limits of Consensual Decision', p. 1279 (formatting altered): 'It is shallow at best to imagine that ... legal choices can be put off: to "not decide" must imply one of two outcomes: (a) tacit permission ... or (b) continuance of an explicit status quo mandate, prohibition or permission.

To see the point's obviousness try to devise a way of effectively postponing a decision on the legality of abortion which can be explained to a pregnant woman. In the choice of candidates for office, either someone is elected or nobody is (yet) elected at any given moment: a final choice can be put off only by accepting an interval of indeterminacy or 'provisional' control which is a very real outcome (consider the Greek colonels or the Portuguese Communists). International agreements can be put off, but a very real and often painful status quo remains to be experienced. These cases suggest a ubiquitous forcing of choice: even when explicit outcomes can be postponed, tacit ones are ipso facto chosen.'

${ }^{47}$ It may seem that under Simple Majority rule ties will be rare and trivial. And so they are, at least in the technical sense introduced above. Although it may make no democratic difference how the issue is decided when there are equal numbers on both sides, it may nonetheless matter enormously to each side how the tied vote is resolved. In that sense, how we resolve ties might be anything but trivial, even under Simple-Majority rule.

${ }^{48}$ The Speaker's case is interestingly different from that of the President of the Senate (the vice-president of the United States), whom the US Constitution (Article I, Section 3) stipulates 'shall have no vote, unless they be equally divided'. 
than one who have such majority, and have an equal number of votes, then the House of Representatives shall immediately chuse by ballot one of them for President ... ${ }^{49}$

The details of those arcane arrangements matter less than their structure, for present purposes. The structure of the decision rule is this: in case of a tie, decide the issue through some other procedure altogether.

\section{TIE-BREAKERS UNDER SYMMETRICAL SPECIAL-MAJORITY RULES}

Here we explore two possible variations on that 'other procedure' strategy for breaking the ties generated by Symmetrical Special-Majority rules. The first, which can be used only in very special circumstances, would be for authorities (perhaps most naturally 'judicial authorities') to decide matters that voting has left formally undecided, by extrapolating further decisions from ones that have already been made under a suitable Symmetrical Special-Majority rule. The second mechanism, which can be used in any federal system, is to let lower levels of government settle issues that are left open by Symmetrical Special-Majority voting at the higher level. ${ }^{50}$

\section{Judicial Extrapolation}

Suppose the legislature has ruled authoritatively on certain propositions, and indeed done so by some very large majority. Suppose, however, it has left certain other matters undecided, and we require some resolution of those issues. Under certain special circumstances, it might be permissible for some authoritative agent (perhaps most naturally judicial authorities) to extrapolate from what has been agreed to by those legislative 'special majorities' to other propositions that are implied by what has been agreed, treating those 'implied' propositions as having the same status as those that had actually been agreed to.

One common principle of extrapolation along these lines, for example, is that 'those who intend an end intend the means strictly necessary to attain that end'. So a legislature that has enacted a statute that assigns powers and responsibilities to the 'Bureau of the Census' will be construed as having endorsed the existence of such a bureau, even if the legislature neglected to stipulate that in the statute itself.

One of the worries surrounding such 'judicial legislation' in general is that the judges might well make laws that are contrary to ones the legislature itself would have enacted. In ordinary circumstances, that is a genuine worry. It is particularly worrisome wherever decisions involving multiple connected propositions are made by Simple-Majority rule. ${ }^{51}$

\footnotetext{
49 Before the Twelfth Amendment, electors cast ballots in this way for two persons, and the person with most votes became president and the person with the next-most votes vice-president. Thus, it was logically possible for two candidates both to be supported by a majority of electors.

${ }^{50}$ We might or might not require that decisions in those other venues be made by the same Symmetrical Special-Majority rules as well. That is not necessary to derive the results discussed below with respect to judicial extrapolation. Under federal subsidiarity rules, it might be the case that the same procedural or epistemic considerations that lead us to demand a special majority of a specific size at the higher level would also apply equally to decision-making at the lower levels; but there might be some reason for thinking that there is something special about the units (member states) doing the voting at the higher level that makes us want to protect minorities among them, in a way that we might not at lower levels where the units (localities) doing the voting enjoy no such morally special status.

${ }^{51}$ Christian List and Philip Pettit, 'Aggregating Sets of Judgments: An Impossibility Result', Economics and Philosophy, 18 (2002), 89-110, and 'Aggregating Sets of Judgments: Two Impossibility Results Compared', Synthese, 140 (2004), 207-35.
} 
To illustrate, imagine three decision makers who each have a certain set of views over three propositions: $P$; ' $P$ implies $Q$ '; and $Q$. The first believes that $P$ is true, that $P$ implies $Q$, and therefore that $Q$ is also true. The second believes that $P$ is true, but does not believe that $P$ implies $Q$, so is consistent in also believing that $Q$ is false. The third accepts that $P$ implies $Q$ but believes that $P$ and $Q$ are both false. Suppose these individuals vote on each of these propositions. A majority (two out of three) hold that $P$ is true; a majority (two out of three) hold that $P$ implies $Q$; and yet a majority (two out of three) hold that $Q$ is false. Were the judiciary to extrapolate from the majority acceptance of $P$ and ' $P$ implies $Q$ ' that $Q$ should also be law, this would be opposite to what a majority vote on $Q$ would have yielded.

Symmetrical Special-Majority voting can assuage those worries. For that to be the case, the 'special majority' supporting each of the component propositions needs to be high: it must be more than $(s-1) / s$ of the individuals, where $s$ is the number of distinct propositions on which decisions are to be made (including basic propositions like $P$ and $Q$ and propositions connecting them like ' $P$ implies $Q$ '). It can be proven that, if propositions (basic and connecting ones) are accepted only if they command a special majority of that size, then there will not be a similarly large special majority against any inferences drawn from the combination of accepted propositions. ${ }^{52}$

Thus, judges can draw, and impose as law, the logical implications of the laws that the legislature has passed by 'special majorities' of the requisite size. (Note well, those implications must be derived by rules of inference that the legislators themselves do - or presumably would - agree to, by a similar-sized 'special majority'.) Judges can do that, confident that their verdicts will not be contrary to the will of any similarly large special majority among the legislature. ${ }^{53}$

\footnotetext{
52 The theorem can be stated as follows. Suppose there are $s$ propositions to be voted on (counting each proposition-negation pair as one proposition), and each individual has a consistent set of views over these propositions. Then proposition-wise (symmetrical) supermajority voting with a supermajority size of more than $(s-1) / s$ of the individuals generates a consistent (but not necessarily complete and deductively closed) collective set of views over the propositions. For a more technical exposition and a proof, see theorem 7 in Christian List, 'A Model of Path-Dependence in Decisions over Multiple Propositions', American Political Science Review, 98 (2004), 495-513. The result has recently been refined by Franz Dietrich and Christian List, 'Judgment Aggregation by Quota Rules', working paper, London School of Economics, http://personal.lse.ac.uk/LIST/PDF-files/ QuotaRules.pdf (accessed 24 January 2004). Proposition-wise (symmetrical) supermajority voting also has an important property in sequential decisions over multiple connected propositions, where (i) different propositions are considered one after the other, (ii) each proposition is decided by some voting rule, but (iii) earlier decisions constrain later ones. If the voting rule is simple-majority voting, then such decision processes may be path-dependent: their outcome may depend on the order in which the propositions are considered. By contrast, if the voting rule is (symmetrical) supermajority voting with a supermajority requirement of more than $(s-1) / s$ of the individuals, then such path-dependence can be avoided. See List, 'A Model of Path-Dependence', and Dietrich and List, 'Judgment Aggregation by Quota Rules'.

${ }^{52}$ So long as $m$ (the legislature's own Symmetrical Special-Majority requirement between the majority and the minority) is similarly large, specifically $m>n\left(2\left({ }^{s-1} / s\right)-1\right)$. To illustrate, if the number of propositions is $s=3$, then $(s-1) / s=2 / 3$, and so in a 100-member legislature $m$ must be at least 34 , corresponding to a majority of at least 67 out of 100 .

${ }^{53}$ Remember the context, here. Judicial extrapolation is being offered as a way of 'breaking ties', filling in matters that are left open by Symmetrical Special-Majority voting in the legislative forum. The scenario in view is one in which the legislature is operating under the rule that 'a special majority of members is required to decide for or against any proposition'; anything less, and the outcome would be considered a 'tie' and left open, legislatively. Judicial extrapolation in those circumstances would therefore not fall foul of the will of the legislature, operating under the rules here in view.
} 
Of course, the requisite 'special majority' $-(s-1) / s$ - increases with the number of propositions $(s)$ involved in the inference. If there are three propositions involved in the inference (including propositions connecting other propositions), those propositions would each have to be endorsed by more than $2 / 3$ of the legislature (or judges have to think they would be so endorsed); if there are four, each by more than $3 / 4$; if eight, each by more than $7 / 8$. Hence this is a mechanism that can plausibly be employed only where the inferences are fairly simple and straightforward, involving few steps. Otherwise the special majority requirement approaches unanimity.

Still, there are such cases. Consider the principle, mentioned above, that 'endorsing the ends implies endorsing the necessary means'. In terms of our three-proposition example above: $P$ is the end; ' $P$ implies $Q$ ' is the proposition that 'if $P$ is the end then $Q$ is a necessary means to that end'; and so $Q$ is the means in question. Since we have three propositions, any special majority requirement of more than $2 / 3$ of the individuals would be sufficient.

\section{Federal Subsidiarity}

A second mechanism for resolving ties generated by Symmetrical Special-Majority voting can be found in the division of decisional responsibilities within a federal system.

To help motivate our proposal here, consider a familiar aspect of appellate court practice. In courts of appeal, it is standard for the judgment of the lower court to be allowed to stand, if the higher court splits evenly on the appeal.

Barry and Hardin discuss that practice and describe it as a violation of May's 'neutrality' condition. ${ }^{54}$ Certainly that rule privileges one outcome over the other: whichever outcome was the one chosen by the lower court. But the 'whichever' in that last sentence is crucial. It is not any particular outcome that is privileged by this rule, as the 'default option' is in the Asymmetrical Special-Majority rule. Instead, what is privileged is a particular decision maker - the lower court. Furthermore, the decision maker thus privileged is not one of the participants in the appeals court (not the senior judge of the panel, for example), which would violate the 'anonymity' condition. What is actually privileged by this rule is a wholly separate decision process, to be used for making the decision when this process yields no determinate choice: when the higher court is tied, the decision of the lower court stands.

Of course, that particular rule actually operates only for the very special case of a literally 'tied' verdict in an appeals court that operates according to Simple-Majority rule. But we propose that model, suitably generalized, as a good strategy for solving the much more frequent 'ties' that can be expected under Symmetrical Special-Majority voting.

The generalized version of this rule, as applied to a federal system (like the European Union, for example), might be this. The higher-level authority can be empowered to act only on the basis of a 'special majority' of the requisite size. There is no 'default' option that wins if no other option commands that number of votes; if no option commands the requisite 'special majority', the higher-level authority has simply not decided in favour of any option. From the point of view of the higher-level authority, it remains a completely open question. But in the context of a federal system operating under a rule of 'subsidiarity' such irresolution at the higher level of authority can simply leave the matter open to determination by authorities at the lower levels.

Determinations of higher-level authorities can still pre-empt determinations of lower-level ones. But where the higher-level authority makes no determinations - here,

54 Barry and Hardin, Rational Man and Irrational Society? p. 297. 
where there is no 'special majority' of the requisite size in favour of any option, at the higher level - the higher-level authority has not pre-empted any decisions by lowerlevel authorities. Under the doctrine of 'subsidiarity', the issue then falls to lower-level authorities (member states, local communities, private individuals) to resolve.

Such strategies resonate particularly powerfully in the EU context, because of its strong traditions of subsidiarity. ${ }^{55}$ But they are common enough even where the central authorities are stronger. The US federal government often intentionally leaves matters that are formally within its jurisdiction open to determination on a state-by-state basis, hoping that the 'laboratory of federalism' might throw up better solutions than any of the federal legislators or bureaucrats can think of for now. The US welfare reforms of 1996 explicitly invited different states to come up with different rules for governing Temporary Assistance to Needy Families, for example. And in its refusal either to endorse a right to physician-assisted suicide or to prohibit it, the US Supreme Court left the matter open for resolution on a state-by-state basis. ${ }^{56}$

Of course, something gets decided, somewhere. Some people win, others lose, in consequence of those decisions. Leaving things undecided at one level just shifts the effective decision point elsewhere.

The point remains, however, that the decision is at least a decision, rather than just the automatic consequence of some arbitrary 'default'. There are reasons for the decision, which people can discuss and debate in those other fora. An Asymmetrical SpecialMajority rule, in contrast, would simply impose some outcome merely by default.

In the course of breaking the 'ties' left by the Symmetrical Special-Majority rule at the higher level, lower-level jurisdictions will often choose different options one from another. Indeed, often they already have.

Such situations pose a compelling case for employing a Symmetrical Special-Majority rule. If different lower-level jurisdictions have already chosen different options, then to let any one of those options serve as the 'default option' under an Asymmetrical Special-Majority rule would arbitrarily favour some jurisdictions and their choices over others. There may well be good grounds for thinking that the higher-level jurisdiction needs to have a 'special majority' of some sort or another to overrule the determinations of the lower-level jurisdictions, in a regime like the EU. But if so, it surely ought to be a Symmetrical Special-Majority requirement that treats all jurisdictions and all options identically. And if the requisite 'special majority' cannot be attained by any of the options at the higher level, then there is a good case to be made in terms of 'subsidiarity' to leave each of the lower-level jurisdictions to determine its own choices for itself. ${ }^{57}$

\section{CONCLUSION}

Majoritarian democrats are ordinarily wary of super-majority requirements. Where we have good grounds for setting the presumption one way rather than another, as we

\footnotetext{
55 Jacques Delors et al., Subsidiarity: The Challenge of Change (Maastricht: European Institute of Public Administration, 1991); George A. Bermann, 'Taking Subsidiarity Seriously: Federalism in the European Community and the United States', Columbia Law Review, 94 (1994), 331-456.

56 The Court's rationale follows the logic set out in Cass R. Sunstein, 'The Right to Die', Yale Law Journal, 106 (1997), 1123-63. The US Supreme Court's actions later that year seem to be acting on that principle: it ruled in 1997 that there is no constitutionally guaranteed right to die, which states are required to respect (in Washington v. Glucksberg and Vacco v. Quill); later that same year, however, the Court also refused to hear a challenge to an Oregon law granting people a right to die (Lee v. Harcleroad, No. 96-1824, certeriori denied).

${ }^{57}$ We are grateful to Dennis Mueller for this observation.
} 
sometimes do, it may be perfectly legitimate to let the outcome often be determined by the defaults built into Asymmetrical Special-Majority rules. But more often there seems to be no good reason for allowing decision rules to favour one outcome over any other in that way, in advance.

The asymmetrical version of the Special-Majority rule is the most familiar form. Were it the only form, unease at the arbitrariness of setting a presumption one way rather than some other (absent any grounds for setting a presumption either way) would incline us towards Simple-Majority rule instead. That is certainly the standard way of avoiding any arbitrary bias in favour of one 'default' option. ${ }^{58}$

The asymmetrical form is not the only possible form of special-majority voting, however. Special-majority requirements can be specified in such a way as to apply equally to all options. Under a Symmetrical Special-Majority rule, no option is privileged as the default option. Arbitrariness is avoided, just as it is under Simple-Majority voting. And something else is achieved as well: procedurally, the special protection that comes with the 'special majority' requirement is afforded to minority interests; epistemically, a higher standard of proof for making decisions is implemented. Such special minority protection or such a special standard of proof may not always be warranted, and, where they are not, Simple-Majority rule works well. But where we have good reasons for thinking that larger-than-bare majorities ought to be required for decisions in any direction, we ought to use a Symmetrical Special-Majority rule instead. And with the symmetrical version of that rule, we can do so in full confidence that no option will be arbitrarily privileged by being the default option.

That decision rule has not heretofore received the attention it deserves, owing perhaps to doubts as to its practical feasibility. Symmetrical Special-Majority rules run the risk of leaving too many things open, which might be undesirable. That practical concern can be addressed, however, if we supplement the Symmetrical Special-Majority rule with other decision procedures, such as venue-shifting options available in the judicial or federal realms.

APPENDIX I: MAJORITARIAN-TYPE RULES AND THEIR PROPERTIES

A Majoritarian-type rule with parameters $k_{-}$and $k_{+}\left(k_{+}>k_{-}\right)$. For any $\mathrm{v}$,

$$
f(v)=\left\{\begin{array}{rl}
1 & \text { if } \sum v_{i} \geq k_{+} \\
0 & \text { if } k_{+}>\sum v_{i}>k_{-} \\
-1 & \text { if } \Sigma v_{i} \leq k_{-}
\end{array} \quad\left(k_{+}>n \text { or } k_{-}<-n \text { are admissible }\right) .\right.
$$

Proposition A1. A voting rule satisfies (U), (A), (M) if and only if it is a Majoritarian-type rule for some values of $k_{+}$and $k_{-}\left(k_{+}>k_{-}\right)$.

Proof. It is easy to see that a Majoritarian-type rule satisfies (U), (A) and (M). Conversely, suppose a voting rule $f$ satisfies (U), (M) and (A).

Step 1. We show that, for any $v, w, \Sigma v_{i} \geq \Sigma w_{i}$ implies $f(v) \geq f(w)$. For each $v$, let $\# v$ denote the number of $1 \mathrm{~s}$ in the profile $\boldsymbol{v}$. Suppose $\Sigma v_{i} \geq \Sigma w_{i}$. Then $\# v \geq \# w$ (since $v_{i}$ and $w_{i}$ only take the values -1 or 1 ).

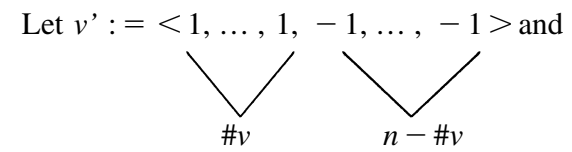

${ }^{58}$ Except to break truly 'trivial' ties.

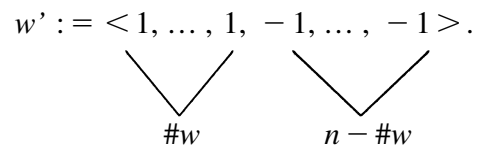

$\sqrt{10}$ 
Since $v^{\prime}$ and $w^{\prime}$ are, respectively, permutations of $v$ and $w$, by (A), we have $f(v)=f\left(v^{\prime}\right)$ and $f(w)=f\left(w^{\prime}\right)$. Since $\# v \geq \# w, v^{\prime} \geq w^{\prime}$. By $(\mathrm{M}), f\left(v^{\prime}\right) \geq f\left(w^{\prime}\right)$. But since $f(v)=f\left(v^{\prime}\right)$ and $f(w)=f\left(w^{\prime}\right)$, we have $f(v) \geq f(w)$, as required.

Step 2. Step 1 implies that there exists an increasing function

$$
g:\{-n,-n+1, \ldots, n-1, n\} \rightarrow\{-1,0,1\}
$$

such that, for any $v, f(v)=g\left(\sum v_{i}\right)$. This implies that there exist $k_{-}, k_{+} \in\{-n,-n+1, \ldots, n-1, n\}$, where $k_{-}<k_{+}$, such that

$$
f(v)=\left\{\begin{array}{rl}
1 & \text { if } \Sigma v_{i} \geq k_{+} \\
0 & \text { if } k_{+}>\sum v_{i}>k_{-} \\
-1 & \text { if } \Sigma v_{i} \leq k_{-}
\end{array} \quad\left(k_{+}>n \text { or } k_{-}<-n \text { are admissible }\right) .\right.
$$

The following propositions state some properties of a Majoritarian-type rule.

Proposition A2. A Majoritarian-type rule never takes the value -1 if $k_{-}<-n$; it never takes the value 1 if $k_{+}>n$; it never takes the value 0 if $k_{+}-k_{-}=1$ or $\left[k_{+}-k_{-}=2\right.$ and $k_{+}$and $n$ are both odd].

Proposition A3. A Majoritarian-type rule satisfies (NT) if and only if either (i) $\left[k_{+}-k_{-}=1\right.$ or $\left[k_{+}-k_{-}=2\right.$ and $k_{+}(\neq 1)$ and $n$ are both odd]] or (ii) $\left[k_{+}=1\right.$ and $\left.k_{-}=-1\right]$.

Case (ii) is the special case of Simple-Majority Voting. Case (i) is the case of Asymmetrical Special-Majority Voting.

Proposition A4. A Majoritarian-type rule satisfies $\left(\mathrm{PV}_{k}\right)$ if and only if $k_{+}>n-2 k$. It satisfies $\left(\mathrm{NV}_{k}\right)$ if and only if $k_{-}<2 k-n$.

APPENDIX II: PROOFS OF THE RESULTS IN SECTION I

Theorem 3 (stated above). A voting rule satisfies (U), (A), (S) and (M) if and only if it is a Symmetrical Special-Majority rule for some parameter $m>0$.

Proof. It is easy to see that, if $f$ is a Symmetrical Special-Majority rule for any value of $m(m>0)$, then $f$ satisfies (U), (A), (S), (M). Suppose, conversely, that $f$ satisfies (U), (A), (S), (M). By proposition A1, there exist $k_{+}$and $k_{-}$(where $k_{+}>k_{-}$) such that, for any $\mathrm{v}$,

$$
f(v)=\left\{\begin{array}{rl}
1 & \text { if } \Sigma v_{i} \geq k_{+} \\
0 & \text { if } k_{+}>\Sigma v_{i}>k_{-} \\
-1 & \text { if } \Sigma v_{i} \leq k_{-}
\end{array} \quad\left(k_{+}>n \text { or } k_{-}<-n \text { are admissible }\right) .\right.
$$

Now, since $f$ satisfies (S), $k_{+}=-k_{-}$. Since $k_{+}>k_{-}$, we must have $k_{+}>0>k_{-}=-k_{+}$. Put $m=k_{+}$. The result follows.

Theorem 1 (May's Theorem) and its corollary can be deduced from Theorem 3, by observing that conditions $\left(\mathrm{VR}_{1}\right)$ and $(\mathrm{NT})$ are each satisfied by a Symmetrical Special-Majority rule if and only if $m=1$ (or $m=2$ and $n$ is even).

Proposition 2 (stated above). If a voting rule satisfies (U), (NT), $\left(\mathrm{PV}_{k_{1}}\right)$ and $\left(\mathrm{NV}_{k_{2}}\right)$, then $k_{1}+k_{2} \geq n$.

Proof. Assume, for a contradiction, that $f$ satisfies the conditions of the proposition with $k_{1}+k_{2}<n$. So we cannot have both $k_{1}=n / 2$ and $k_{2}=n / 2$. Without loss of generality, assume that $k_{1} \neq n / 2$. Consider a profile $v$ where $v_{1}, \ldots, v_{k_{1}}=-1$, and $v_{k_{1}+1}, \ldots, v_{n}=1$. Then $\left|\left\{i \in N: v_{i}=-1\right\}\right|=k_{1}$, and so, by $\left(\mathrm{PV}_{k_{1}}\right)$, $f(v) \neq 1$. Also, $\left|\left\{i \in N: v_{i}=1\right\}\right|=n-k_{1}>k_{2}\left(\right.$ since $\left.k_{1}+k_{2}<n\right)$ and so, by $\left(\mathrm{NV}_{k_{2}}\right), f(v) \neq-1$. Hence $f(v)=0$. By (NT), this implies $\Sigma v_{i}=0$. But $\Sigma v_{i}=n-2 k_{1} \neq 0$, a contradiction. Hence $k_{1}+k_{2} \geq n$.

Proposition 4 (stated above). Suppose a voting rule satisfies (U) and (S). Then, for any $k$, it satisfies $\left(\mathrm{PV}_{k}\right)$ if and only if it satisfies $\left(\mathrm{NV}_{k}\right)$.

Proof. This proposition follows immediately from condition (S).

The conjunction of Propositions 2 and 4 immediately entails Theorem 2. 
Proposition 1 (stated above). A voting rule satisfies (U), (NT), (S) and $\left(\mathrm{PV}_{n / 2}\right)$ (and $\left(\mathrm{NV}_{n / 2}\right)$ ) if and only if it is Simple-Majority rule.

Proof. If $f$ is Simple-Majority rule, $f$ clearly satisfies the relevant conditions. We prove the reverse implication. Suppose $f$ satisfies $(\mathrm{U}),(\mathrm{NT}),(\mathrm{S})$ and $\left(\mathrm{PV}_{n / 2}\right)$. Suppose $\Sigma v_{i}<0$. This implies $\mid\{i \in N$ : $\left.v_{i}=-1\right\} \mid \geq n / 2$, and hence, by $\left(\mathrm{PV}_{n / 2}\right), f(v) \neq 1$. But, since $\Sigma v_{i}<0$, (NT) implies $f(v) \neq 0$, and therefore $f(v)=-1$. By (S), if $\Sigma v_{i}>0$, this implies that $f(v)=1$. If $\Sigma v_{i}=0$, we must have $\left|\left\{i \in N: v_{i}=-1\right\}\right|=n / 2$. By $\left(\mathrm{PV}_{n / 2}\right), f(v) \neq 1$. But by (S), we must also have $f(v) \neq-1$, i.e. $f(v)=0$. Hence $f$ is Simple-Majority rule.

Propositions 3 and 5 follow immediately from Proposition A4.

Proposition 6 follows from the definition of a Symmetrical Special-Majority rule. Note that, for any $m$, a Symmetrical Special-Majority rule with parameter $m$ is equivalent to a rule with parameter $m^{*}$, where

$$
m^{*} \text { is }\left\{\begin{array}{l}
\text { the smallest even number greater than or equal to } m \text { if } n \text { is even } \\
\text { the smallest odd number greater than or equal to } m \text { if } n \text { is odd. }
\end{array}\right.
$$

Proposition 7 follows immediately from Theorem 3 and Propositions 5 and 6.

Proposition 8 (stated above). If a Symmetrical Special-Majority rule satisfies both $\left(\mathrm{PV}_{k_{1}}\right)$ and $\left(\mathrm{VR}_{k_{2}}\right)$, then $k_{2}>n-2 k_{1}$.

Proof. Suppose $f$ is a Symmetrical Special-Majority rule with parameter $m$. Without loss of generality we can assume that $m$ is even if $n$ is even, and odd if $n$ is odd. By proposition $5, m>n-2 k_{1}$. By Proposition $6, m<k_{2}+2$. Note that $n-2 k_{1}$ is even if and only if $n$ is even. So, when $n$ is even, the smallest even number greater than $n-2 k_{1}$ is $n-2 k_{1}+2$. Likewise, when $n$ is odd, the smallest odd number greater than $n-2 k_{1}$ is $n-2 k_{1}+2$. So $m \geq n-2 k_{1}+2$. But, since $m<k_{2}+2$, we must have $k_{2}+2>m \geq n-2 k_{1}+2$, i.e. $k_{2}>n-2 k_{1}$.

APPENDIX III: PROOFS OF THE RESULTS IN SECTION II

Theorem 4 (restated). For any standard of proof parameter $c$ significantly greater than $1 / 2$, there exists no voting rule satisfying $(\mathrm{U}),\left(\mathrm{NT}^{*}\right),\left(\mathrm{PP}_{c}\right)$ and $\left(\mathrm{NP}_{c}\right)$.

Proof. Suppose $f$ is a voting rule satisfying $(\mathrm{U}),\left(\mathrm{NT}^{*}\right),\left(\mathrm{PP}_{c}\right)$ and $\left(\mathrm{NP}_{c}\right)$.

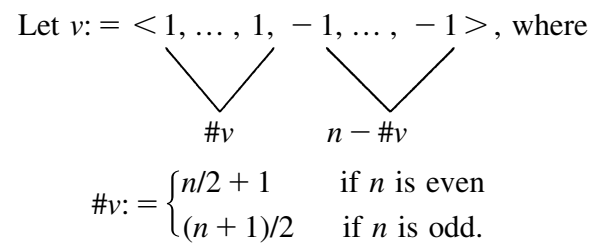

Then $\Sigma v_{i}=2$ if $n$ is even, and $\Sigma v_{i}=1$ if $n$ is odd. By a formula by Condorcet, ${ }^{59} \operatorname{Pr}(X=1 \mid V=v)=$ $p^{\Sigma v_{i}} /\left(p^{\Sigma v_{i}}+(1-p)^{\Sigma v_{i}}\right)$. So $\left.\operatorname{Pr}(X=1 \mid V=v)=p^{2} /\left(p^{2}+(1-p)^{2}\right)\right)$ if $n$ is even, and $\operatorname{Pr}(X=1 \mid V=v)=p$ if $n$ is odd. Note that $\operatorname{Pr}(X=1 \mid V=v) \neq 1 / 2$. Since $f$ satisfies $\left(\mathrm{NT}^{*}\right)$, we must have $f(v) \neq 0$. So $f(v)=1$ or $f(v)=-1$. Since $f$ satisfies $\left(\operatorname{PP}_{c}\right)$, if $f(v)=1$, then $\operatorname{Pr}(X=1 \mid V=v)>c$; so $\left.c<p^{2} /\left(p^{2}+(1-p)^{2}\right)\right)$ if $n$ is even, and $c<p$ if $n$ is odd. Since $f$ satisfies $\left(\mathrm{NP}_{c}\right)$, if $f(v)=-1$, then $\operatorname{Pr}(X=-1 \mid V=v)>c$, i.e. $1-\operatorname{Pr}(X=1 \mid V=v)>c$; so $\left.\left.c<1-p^{2} /\left(p^{2}+(1-p)^{2}\right)\right)<p^{2} /\left(p^{2}+(1-p)^{2}\right)\right)$ if $n$ is even, and $c<1-p<p$ if $n$ is odd. Therefore $c$ is not significantly greater than $1 / 2$, in the technical sense defined above.

Proposition 9 (restated). In Condorcet's model, a voting rule satisfies $(\mathrm{U}),\left(\mathrm{PP}_{1 / 2}\right)$ and $\left(\mathrm{NP}_{1 / 2}\right)$ if and only if it is Simple-Majority Voting.

${ }^{59}$ List, 'On the Significance of the Absolute Margin'. 
Proof. It is easy to check that Simple-Majority Voting satisfies $(\mathrm{U}),\left(\mathrm{PP}_{1 / 2}\right)$ and $\left(\mathrm{NP}_{1 / 2}\right)$ (under Condorcet's assumptions). Suppose $f$ satisfies $(\mathrm{U}),\left(\mathrm{PP}_{1 / 2}\right)$ and $\left(\mathrm{NP}_{1 / 2}\right)$. Let $v$ be any profile. $\mathrm{By}\left(\mathrm{PP}_{1 / 2}\right)$ and $\left(\mathrm{NP}_{1 / 2}\right)$,

$$
\begin{aligned}
& f(v)=1 \text { if and only if } \operatorname{Pr}(X=1 \mid V=v)>1 / 2, \\
& f(v)=-1 \text { if and only if } \operatorname{Pr}(X=-1 \mid V=v)>1 / 2 .
\end{aligned}
$$

By (U), we must therefore have $f(v)=0$ if and only if $\operatorname{Pr}(X=1 \mid V=v)=1 / 2$.

By Condorcet's formula, ${ }^{60} \operatorname{Pr}(X=1 \mid V=v)=p^{\Sigma v_{i}} /\left(p^{\Sigma v_{i}}+(1-p)^{\Sigma v_{i}}\right) \quad$ and $\quad \operatorname{Pr}(X=-1 \mid V=v)=$ $1-p^{\Sigma v_{i}} /\left(p^{\Sigma v_{i}}+(1-p)^{\Sigma v_{i}}\right)$. So

$$
\begin{array}{ll}
f(v)=1 \text { if and only if } & p^{\Sigma v_{i}} /\left(p^{\Sigma v_{i}}+(1-p)^{\Sigma v_{i}}\right)>1 / 2, \text { and } \\
f(v)=-1 \text { if and only if } & 1-p^{\Sigma v_{i}} /\left(p^{\Sigma v_{i}}+(1-p)^{\Sigma v_{i}}\right)>1 / 2, \\
& \text { i.e. } p^{\Sigma v_{i}} /\left(p^{\Sigma v_{i}}+(1-p)^{\Sigma v_{i}}\right)<1 / 2 .
\end{array}
$$

But

$$
\begin{array}{ll}
p^{\Sigma v_{i}} /\left(p^{\Sigma v_{i}}+(1-p)^{\Sigma v_{i}}\right)>1 / 2 \text { if and only if } & p^{\Sigma v_{i}}>(1-p)^{\Sigma v_{i}}, \\
& \text { i.e. } \Sigma v_{i}>0(\text { since } p>1 / 2) \\
p^{\Sigma v_{i}} /\left(p^{\Sigma v_{i}}+(1-p)^{\Sigma v_{i}}\right)<1 / 2 \text { if and only if } & p^{\Sigma v_{i}}<(1-p)^{\Sigma v_{i}}, \\
& \text { i.e. } \Sigma v_{i}<0(\text { since } p>1 / 2) .
\end{array}
$$

Finally, $p^{\Sigma v_{i}} /\left(p^{\Sigma v_{i}}+(1-p)^{\Sigma v_{i}}\right)=1 / 2$ if and only if $p^{\Sigma v_{i}}=(1-p)^{\Sigma v_{i}}$, i.e. $\Sigma v_{i}=0$ (since $\left.p \neq 1 / 2\right)$. We conclude that $f$ is Simple-Majority Voting.

Proposition 10 (restated). Suppose a voting rule satisfies (U) and (NT*), and suppose $c_{1}$ is significantly greater than $1 / 2$. If the voting rule satisfies $\left(\mathrm{PP}_{c_{1}}\right)$, then it does not satisfy $\left(\mathrm{NP}_{c_{2}}\right)$ for any $c_{2} \geq 1 / 2$; and if it satisfies $\left(\mathrm{NP}_{c_{1}}\right)$, then it does not satisfy $\left(\mathrm{PP}_{c_{2}}\right)$ for any $c_{2} \geq 1 / 2$.

Proof. Suppose $f$ satisfies $(\mathrm{U}),\left(\mathrm{NT}^{*}\right)$ and $\left(\mathrm{PP}_{c_{1}}\right)$, where $c_{1}$ is significantly greater than $1 / 2$. Suppose further that $f$ satisfies $\left(\mathrm{NP}_{c_{2}}\right)$ for some $c_{2} \geq 1 / 2$. Define $v$ as in the proof of theorem 4. Then $c_{1} \geq \operatorname{Pr}(X=1 \mid V=v)>1 /$ 2. By $\left(\mathrm{PP}_{c_{1}}\right), f(v) \neq 1$. By $(\mathrm{NT} *), f(v) \neq 0$. But $\operatorname{Pr}(X=-1 \mid V=v)=1-\operatorname{Pr}(X=1 \mid V=v)<1 / 2 \leq c_{2}$, so by $\left(\mathrm{NP}_{c_{2}}\right), f(v) \neq-1$. This contradicts $(\mathrm{U})$. The proof that if $f$ satisfies $\left(\mathrm{NP}_{c_{1}}\right)$ then $f$ does not satisfy $\left(\mathrm{PP}_{c_{2}}\right)$ for any $c_{2} \geq 1 / 2$ is analogous.

Proposition 11 (restated). Let $c \geq 1 / 2$. A voting rule satisfies $(\mathrm{U}),\left(\mathrm{PP}_{c}\right)$ and $\left(\mathrm{NP}_{c}\right)$ if and only if it is a Symmetrical Special-Majority rule, where the parameter $m$ is the smallest integer strictly greater than $\log (1 / c-1) / \log \left({ }^{1} / p-1\right)$.

Proof. Suppose $f$ satisfies $(\mathrm{U}),\left(\mathrm{PP}_{c}\right)$ and $\left(\mathrm{NP}_{c}\right)$, where $m$ is the smallest integer above $\log \left({ }^{1} / c-1\right) /$ $\log (1 / p-1))$. Then

$$
\begin{aligned}
& f(v)=1 \text { if and only if } \operatorname{Pr}(X=1 \mid V=v)>c, \\
& f(v)=-1 \text { if and only if } \operatorname{Pr}(X=-1 \mid V=v)>c .
\end{aligned}
$$

By (U), we must therefore have $f(v)=0$ if and only if $c \geq \operatorname{Pr}(X=1 \mid V=v) \geq 1-c$.

By a theorem in an earlier paper by List, ${ }^{61}$

$$
\left.\operatorname{Pr}(X=1 \mid V=v)>c \text { if and only if } \quad \sum v_{i}>\log \left(1 /{ }_{c}-1\right) / \log (1 / p-1)\right),
$$

i.e. $\Sigma v_{i} \geq m$, with $m$ as defined above.

Similarly, $\operatorname{Pr}(X=-1 \mid V=v)>c$ if and only if $\left.\quad-\Sigma v_{i}>\log (1 / c-1) / \log (1 / p-1)\right)$,

i.e. $\Sigma v_{i} \leq-m$, with $m$ as before.

Finally, $c \geq \operatorname{Pr}(X=1 \mid V=v) \geq 1-c$ if and only if $m>\sum v_{i}>-m$. We conclude that $f$ is a Symmetrical Special-Majority rule with parameter $m$. It is easy to check that, if $f$ is a Symmetrical Special-Majority rule with parameter $m$, then $f$ satisfies $(\mathrm{U}),\left(\mathrm{PP}_{c}\right)$ and $\left(\mathrm{NP}_{c}\right)$ with $m$ as defined above.

${ }^{60}$ List, 'On the Significance of the Absolute Margin'.

${ }^{61}$ List, 'On the Significance of the Absolute Margin'. 
APPENDIX IV: A VERSION OF THE EPISTEMIC CASE IN TERMS OF THE DEGREE OF SUPPORT

Again we make Condorcet's assumptions, except that no assumption about prior probabilities is required here. The degree of support some evidence $E$ gives to some hypothesis $H$ is defined to be $l(H$, $E):=\log \left(\operatorname{Pr}(E \mid H) / \operatorname{Pr}(E \mid \neg H){ }^{62}\right.$ The degree of support the voting pattern $V=v$ gives to the hypothesis $X=1$ is $l(X=1, V=v)=\sum v_{i} \log (p /(1-p))$. The degree of support the voting pattern $V=v$ gives to the hypothesis $X=-1$ is $l(X=-1, V=v)=\Sigma v_{i} \log ((1-p) / p)=-\sum v_{i} \log (p /(1-p))$.

Now a classical (non-Bayesian) way of stating the standard of proof conditions is as follows:

A standard of proof of $c$ for positive decisions $\left(P P^{*}{ }_{c}\right)$. For any profile $v, f(v)=1$ if and only if $l(X=1$, $V=v)>c$.

A standard of proof of c for negative decisions $\left(N P^{*}{ }_{c}\right)$. For any profile $v, f(v)=-1$ if and only if $l(X=-1$, $V=v)>c$.

The standard of proof corresponding to Simple-Majority rule is $c=0$. We can now state corollaries of Theorem 4 and Propositions 9 to 11 .

Proposition 9*. In Condorcet's model, a voting rule satisfies $(\mathrm{U}),\left(\mathrm{PP} *_{0}\right)$ and $\left(\mathrm{NP} *_{0}\right)$ if and only if it is Simple-Majority rule.

The classical (non-Bayesian) statement of no non-trivial ties, then, is

No non-trivial ties (NT**). For any profile $v, f(v)=0$ implies $l(X=1, V=v)=l(X=-1, V=v)=0$.

Clearly, Simple-Majority Voting satisfies (NT**).

Let us say that the threshold $c$ is significantly greater than 0 if

$$
c \geq \begin{cases}\log (p /(1-p)) & \text { if } n \text { is odd } \\ 2 \log (p /(1-p)) & \text { if } n \text { is even. }\end{cases}
$$

Theorem $4 *$. For any standard of proof parameter $c$ significantly greater than 0 , there exists no voting rule satisfying $(\mathrm{U}),\left(\mathrm{NT}^{* *}\right),\left(\mathrm{PP}^{*}{ }_{c}\right)$ and $\left(\mathrm{NP}^{*}{ }_{c}\right)$.

Proposition 10*. Suppose a voting rule satisfies (U) and (NT**), and suppose $c_{1}$ is significantly greater than 0 . If the voting rule satisfies $\left(\mathrm{PP} *{ }_{c_{1}}\right)$, then it does not satisfy $\left(\mathrm{NP}^{*}{ }_{c_{2}}\right)$ for any $c_{2} \geq 0$; and if it satisfies $\left(\mathrm{NP}_{c_{1}}\right)$, then it does not satisfy $\left(\mathrm{PP}_{c_{2}}\right)$ for any $c_{2} \geq 0$.

Proposition $11 *$ Let $c \geq 0$. A voting rule satisfies $(\mathrm{U}),\left(\mathrm{PP}_{c}\right)$ and $\left(\mathrm{NP}_{c}\right)$ if and only if it is Symmetrical Special-Majority Voting, where $m$ is the smallest integer strictly greater than $c / \log (p /(1-p))$.

The case $c=0$ corresponds to Simple-Majority rule. The case $c$ significantly greater than 0 but less than $n \log (p /(1-p))$ corresponds to a Special-Majority rule. The case $c$ greater than or equal to $n \log (p /(1-p))$ corresponds to Imposed Indifference.

62 Branden Fitelson, 'A Bayesian Account of Independent Evidence with Applications', Philosophy of Science, 68 (Proceedings) (2001), S123-40. 
